L. Escauriaza · C. E. Kenig · G. Ponce · L. Vega

\title{
Hardy's uncertainty principle, convexity and Schrödinger evolutions
}

Received February 12, 2008

\begin{abstract}
We prove the logarithmic convexity of certain quantities, which measure the quadratic exponential decay at infinity and within two characteristic hyperplanes of solutions of Schrödinger evolutions. As a consequence we obtain some uniqueness results that generalize (a weak form of) Hardy's version of the uncertainty principle. We also obtain corresponding results for heat evolutions.
\end{abstract}

Keywords. Schrödinger evolutions

\section{Introduction}

In this paper we continue the study initiated in [11] and [2] on unique continuation properties of solutions of Schrödinger evolutions

$$
i \partial_{t} u+\Delta u=V(x, t) u \quad \text { in } \mathbb{R}^{n} \times[0,1] .
$$

The goal is to obtain sufficient conditions on a solution $u$, the potential $V$ and the behavior of the solution at two different times, $t_{0}=0$ and $t_{1}=1$, which guarantee that $u \equiv 0$ in $\mathbb{R}^{n} \times[0,1]$.

One of our motivations comes from a well known result due to G. H. Hardy [16, pp. 131] (see also [1] for a recent survey on this topic), which concerns the decay of a function $f$ and its Fourier transform,

$$
\hat{f}(\xi)=(2 \pi)^{-n / 2} \int_{\mathbb{R}^{n}} e^{-i \xi \cdot x} f(x) d x
$$

L. Escauriaza: Departamento de Matemáticas, UPV/EHU, Apto. 644, 48080 Bilbao, Spain; e-mail: luis.escauriaza@ehu.es

C. E. Kenig: Department of Mathematics, University of Chicago, Chicago, IL. 60637, USA; e-mail: cek@math.uchicago.edu

G. Ponce: Department of Mathematics, University of California, Santa Barbara, CA 93106, USA; e-mail: ponce@math.ucsb.edu

L. Vega: Departamento de Matemáticas, UPV/EHU, Apto. 644, 48080 Bilbao, Spain;

e-mail:mtpvegol@lg.ehu.es

Mathematics Subject Classification (2000): Primary 35B05; Secondary 35B60 
and states:

If $f(x)=O\left(e^{-|x|^{2} / \beta^{2}}\right), \hat{f}(\xi)=O\left(e^{-4|\xi|^{2} / \alpha^{2}}\right)$, and $\alpha \beta<4$, then $f \equiv 0$. Also, if $\alpha \beta=4$, then $f$ is a constant multiple of $e^{-|x|^{2} / \beta^{2}}$,

This result can be rewritten in terms of the free solution of the Schrödinger equation in $\mathbb{R}^{n} \times(0,+\infty), i \partial_{t} u+\Delta u=0$, with initial data $f$,

$$
u(x, t)=(4 \pi i t)^{-n / 2} \int_{\mathbb{R}^{n}} e^{i|x-y|^{2} / 4 t} f(y) d y=(2 \pi i t)^{-n / 2} e^{i|x|^{2} / 4 t} e^{\widehat{i|\cdot|^{2} / 4 t}} f\left(\frac{x}{2 t}\right)
$$

in the following way:

$$
\text { If } u(x, 0)=O\left(e^{-|x|^{2} / \beta^{2}}\right), u(x, T)=O\left(e^{-|x|^{2} / \alpha^{2}}\right) \text {, and } \alpha \beta<4 T \text {, then } u \equiv 0 . \text { Also, }
$$
if $\alpha \beta=4 T$, then $u$ has as initial data a constant multiple of $e^{-\left(1 / \beta^{2}+i / 4 T\right)|y|^{2}}$.

The corresponding result in terms of $L^{2}$-norms and established in [15] is the following:

If $e^{|x|^{2} / \beta^{2}}$ f and $e^{4|\xi|^{2} / \alpha^{2}} \widehat{f}$ are in $L^{2}\left(\mathbb{R}^{n}\right)$ and $\alpha \beta \leq 4$, then $f \equiv 0$.

If $e^{|x|^{2} / \beta^{2}} u(x, 0)$ and $e^{|\xi|^{2} / \alpha^{2}} u(x, T)$ are in $L^{2}\left(\mathbb{R}^{n}\right)$ and $\alpha \beta \leq 4 T$, then $u \equiv 0$.

In our previous paper [2] we proved a uniqueness result in this direction for potentials which satisfy

$$
\lim _{R \rightarrow+\infty} \int_{0}^{1}\|V(t)\|_{L^{\infty}\left(\mathbb{R}^{n} \backslash B_{R}\right)} d t=0 .
$$

More precisely, we proved that the only solution to 1.1$]$ in $C\left([0,1], H^{2}\left(\mathbb{R}^{n}\right)\right)$ which together with its gradient decays faster than any quadratic exponential at times 0 and 1 is the zero solution when $V$ is bounded in $\mathbb{R}^{n} \times[0,1]$, (1.2) holds, and $\nabla_{x} V$ is in $L_{t}^{1} L_{x}^{\infty}\left(\mathbb{R}^{n} \times[0,1]\right)$. This linear result was then applied to show that two regular solutions $u_{1}$ and $u_{2}$ of non-linear equations of the type

$$
i \partial_{t} u+\Delta u=F(u, \bar{u}) \quad \text { in } \mathbb{R}^{n} \times[0,1],
$$

for very general non-linearities $F$, must agree in $\mathbb{R}^{n} \times[0,1]$ when $u_{1}-u_{2}$ and its gradient decay faster than any quadratic exponential at times 0 and 1 . This replaced the assumption that the solutions coincide on large subdomains of $\mathbb{R}^{n}$ at two different times, which was previously studied in [11, 7], and showed that weaker variants of Hardy's theorem hold even in the context of non-linear Schrödinger evolutions.

Our main result in this paper is the following one.

Theorem 1. Assume that $u$ in $C\left([0,1], L^{2}\left(\mathbb{R}^{n}\right)\right)$ satisfies

$$
\partial_{t} u=i(\Delta u+V(x, t) u) \quad \text { in } \mathbb{R}^{n} \times[0,1],
$$

$\alpha$ and $\beta$ are positive, $\alpha \beta<2,\left\|e^{|x|^{2} / \beta^{2}} u(0)\right\|_{L^{2}\left(\mathbb{R}^{n}\right)}$ and $\left\|e^{|x|^{2} / \alpha^{2}} u(1)\right\|_{L^{2}\left(\mathbb{R}^{n}\right)}$ are both finite, the potential $V$ is bounded, and either $V(x, t)=V_{1}(x)+V_{2}(x, t)$ with $V_{1}$ realvalued and

$$
\sup _{[0,1]}\left\|e^{\frac{|x|^{2}}{(\alpha t+\beta(1-t))^{2}}} V_{2}(t)\right\|_{L^{\infty}\left(\mathbb{R}^{n}\right)}<+\infty,
$$

or $\lim _{R \rightarrow+\infty}\|V\|_{L^{1}\left([0,1], L^{\infty}\left(\mathbb{R}^{n} \backslash B_{R}\right)\right)}=0$. Then $u \equiv 0$. 
As a direct consequence of Theorem 1 we get the following straightforward result concerning the uniqueness of solutions for non-linear equations of the form 1.3 .

Theorem 2. Let $u_{1}$ and $u_{2}$ be $C\left([0,1], H^{k}\left(\mathbb{R}^{n}\right)\right)$ strong solutions of the equation (1.3) with $k \in \mathbb{Z}^{+}, k>n / 2, F: \mathbb{C}^{2} \rightarrow \mathbb{C}, F \in C^{k}$, and $F(0)=\partial_{u} F(0)=\partial_{\bar{u}} F(0)=0$. If there are $\alpha$ and $\beta$ positive with $\alpha \beta<2$ such that

$$
e^{|x|^{2} / \beta^{2}}\left(u_{1}(0)-u_{2}(0)\right) \quad \text { and } e^{|x|^{2} / \alpha^{2}}\left(u_{1}(1)-u_{2}(1)\right)
$$

are in $L^{2}\left(\mathbb{R}^{n}\right)$, then $u_{1} \equiv u_{2}$.

Notice that the condition $\alpha \beta<2$ is independent of the size of the potential or the dimension and that we do not assume any decay of the gradient neither of the solutions or of time-independent potentials or any regularity of the potentials.

Our improvement over the results of [2] comes from a better understanding of the solutions to 1.1 which have a Gaussian decay. We started the study of this particular type of solutions in our recent work [3], where we consider free waves (i.e. $V(x, t)=0$ in (1.1) and among other results we proved the following:

Assume that $u$ in $C\left([0,1], L^{2}\left(\mathbb{R}^{n}\right)\right)$ is a solution of

$$
\partial_{t} u-i \Delta u=0 \quad \text { in } \mathbb{R}^{n} \times[0,1],
$$

and that $\left\|e^{\gamma|x|^{2}} u(0)\right\|,\left\|e^{\gamma|x|^{2}} u(1)\right\|$ are both finite. Set $f=e^{\gamma|x|^{2}} u$ and $H(t)=(f, f)$. Then $\log H(t)$ is a convex function.

The proof of Theorem 1 relies first on extending the above convexity properties to the non-free case, and secondly on a modification of the definition of the function $H$ as follows: for $e_{1}=(1,0, \ldots, 0), R>0$ and $0<\mu<\gamma$ set

$$
f=e^{\mu\left|x+R t(1-t) e_{1}\right|^{2}} u
$$

and $H(t)=(f, f)$. Then it is easy to prove at a formal level that

$$
\partial_{t}^{2} \log H(t) \geq-\frac{R^{2}}{4 \mu}
$$

Therefore $H(t) e^{-R^{2} t(1-t) / 8 \mu}$ is logarithmically convex in $[0,1]$ and

$$
H(t) \leq H(0)^{1-t} H(1)^{t} e^{R^{2} t(1-t) / 8 \mu} .
$$

Taking $t=1 / 2$ and letting $\mu$ increase towards $\gamma$, we have

$$
\int e^{2 \gamma\left|x+(R / 4) e_{1}\right|^{2}}|u(1 / 2)|^{2} d x \leq\left\|e^{\gamma|x|^{2}} u(0)\right\|\left\|e^{\gamma|x|^{2}} u(1)\right\| e^{R^{2} / 32 \gamma} .
$$

Thus,

$$
\int_{B_{\epsilon R / 4}}|u(1 / 2)|^{2} d x \leq\left\|e^{\gamma|x|^{2}} u(0)\right\|\left\|e^{\gamma|x|^{2}} u(1)\right\| e^{R^{2}\left(1-4 \gamma^{2}(1-\epsilon)^{2}\right) / 32 \gamma}
$$

when $0<\epsilon<1$, which implies that $u \equiv 0$ by letting $R$ tend to infinity, when $\gamma>1 / 2$. 
The path that goes from the formal level to a rigorous one is not an easy one. In fact, in Section 6 we will give explicit examples of functions $H(t)$ such that $\log H$ is formally convex but the corresponding inequalities lead to false statements. Therefore most of this paper is devoted to making the above argument rigorous. The starting point is to prove similar properties to those obtained in [4] for free solutions. One of the results we get is the following one.

Theorem 3. Assume that $u$ in $C\left([0,1], L^{2}\left(\mathbb{R}^{n}\right)\right)$ satisfies

$$
\partial_{t} u=i(\Delta u+V(x, t) u) \quad \text { in } \mathbb{R}^{n} \times[0,1],
$$

$V=V_{1}(x)+V_{2}(x, t), V_{1}$ is real-valued, $\left\|V_{1}\right\|_{\infty} \leq M_{1}$, and there are positive numbers $\alpha$ and $\beta$ such that

$$
\left\|e^{|x|^{2} / \beta^{2}} u(0)\right\|,\left\|e^{|x|^{2} / \alpha^{2}} u(1)\right\|, \sup _{[0,1]}\left\|e^{\frac{|x|^{2}}{(\alpha t+(1-t) \beta)^{2}}} V_{2}(t)\right\|_{\infty}<+\infty .
$$

Then $\left\|e^{\frac{|x|^{2}}{(\alpha t+(1-t) \beta)^{2}}} u(t)\right\|^{\alpha t+(1-t) \beta}$ is "logarithmically convex" in $[0,1]$ and there is $N=$ $N(\alpha, \beta)$ such that

$$
\begin{aligned}
& \qquad e^{\frac{|x|^{2}}{(\alpha t+(1-t) \beta)^{2}}} u(t)\left\|\leq e^{N\left(M_{1}+M_{2}+M_{1}^{2}+M_{2}^{2}\right)}\right\| e^{|x|^{2} / \beta^{2}} u(0)\left\|\frac{\beta(1-t)}{\alpha t+\beta(1-t)}\right\| e^{|x|^{2} / \alpha^{2}} u(1) \| \frac{\alpha t}{\alpha t+\beta(1-t)} \\
& \text { when } 0 \leq t \leq 1 \text { and } M_{2}=\sup _{[0,1]}\left\|e^{\frac{|x|^{2}}{(\alpha t+\beta(1-t))^{2}}} V_{2}(t)\right\|_{\infty} e^{2 \sup _{[0,1]}\left\|\operatorname{Im} V_{2}(t)\right\|_{\infty}} \text {. Moreover, } \\
& \qquad \begin{aligned}
\left\|\sqrt{t(1-t)} e^{\frac{|x|^{2}}{(\alpha t+(1-t) \beta)^{2}}} \nabla u\right\|_{L^{2}\left(\mathbb{R}^{n} \times[0,1]\right)} \\
\leq N e^{N\left(M_{1}+M_{2}+M_{1}^{2}+M_{2}^{2}\right)}\left[\left\|e^{|x|^{2} / \beta^{2}} u(0)\right\|+\left\|e^{|x|^{2} / \alpha^{2}} u(1)\right\|\right] .
\end{aligned}
\end{aligned}
$$

In order to prove this theorem we have to approximate the solution using some artificial diffusion. The corresponding results are interesting in themselves and can be found in Section 2. As a byproduct we get examples of solutions to 1.1 which have Gaussian decay, when the potential $V$ is time independent. It is enough to consider as initial data the solution, at say time one, of the corresponding heat equation that at time zero is a Gaussian. This property was already established in [4] for free solutions, and it turned out to be a characterization of those Gaussian solutions. It would be interesting to prove similar characterizations for variable coefficient Hamiltonians. Also in Section 2 we give an abstract result, Lemma 2 that shows how to get logarithmic convexity properties from the positivity of some specific commutators. It turns out that these commutators are the same as the ones that appear in the proof of the $L^{2}$-Carleman estimates we used in our previous paper [2]. In fact, the weight $\mu\left|x+\operatorname{Re}_{1} t(1-t)\right|^{2}$ that appears in (1.4) is a refinement of the ones used in [2].

We are indebted to E. Zuazua for pointing out the application of Hardy's uncertainty principle to prove the following optimal decay result for solutions of the free heat equation (see also [10, Section 5]): 
If $f$ and $e^{|x|^{2} / \delta^{2}} e^{\Delta} f$ are in $L^{2}\left(\mathbb{R}^{n}\right)$ for some $\delta \leq 2$, then $f \equiv 0$.

In fact, applying Hardy's uncertainty principle to $e^{\Delta} f$ shows that $e^{|x|^{2} / \delta^{2}} e^{\Delta} f$ and $e^{4|\xi|^{2} / 2^{2}} \widehat{e^{\Delta} f}=\widehat{f}$ are in $L^{2}\left(\mathbb{R}^{n}\right)$, and $2 \delta \leq 4$ implies $e^{\Delta} f \equiv 0$. Then backward uniqueness arguments (see for instance [13, Chapter 3, Theorem 11] or [5, Chapter 3]) show that $f \equiv 0$. Here, we prove the following weaker extension of this result for parabolic operators with variable coefficients.

Theorem 4. Let $u$ in $L^{\infty}\left([0,1], L^{2}\left(\mathbb{R}^{n}\right)\right) \cap L^{2}\left([0, T], H^{1}\left(\mathbb{R}^{n}\right)\right)$ satisfy

$$
\left\{\begin{array}{l}
\partial_{t} u=\Delta u+V(x, t) u \quad \text { in } \mathbb{R}^{n} \times(0,1], \\
u(0)=f
\end{array}\right.
$$

where $V$ is bounded in $\mathbb{R}^{n} \times[0,1]$, and assume that $f$ and $e^{|x|^{2} / \delta^{2}} u(1)$ are in $L^{2}\left(\mathbb{R}^{n}\right)$ for some $\delta<1$. Then $f \equiv 0$ in $\mathbb{R}^{n}$.

It is natural to expect that the Hardy uncertainty principle holds for Schrödinger and heat evolutions with bounded potentials and with parameters $\alpha, \beta$ or $\delta$ satisfying the condition of the free case.

In the sketch of the proof of Theorem 1 that we have given above we have assumed that $\alpha=\beta$. That one can easily reduce to this case is proved in Section 3 using the so called conformal transformation or Appell transform. In Section 4 we prove Theorem 3 . in Section 5 we give the proof of Theorem 1 , in Section 6 we give some examples of some misleading convex functions, and in Section 7 we prove Theorem 4

\section{A few lemmas}

In the following,

$$
(f, g)=\int_{\mathbb{R}^{n}} f \bar{g} d x, \quad\|f\|^{2}=(f, f), \quad f^{+}=\max \{f, 0\}
$$

and $\|f\|_{\infty}$ denotes the $L^{\infty}$-norm of $f$ over $\mathbb{R}^{n}$.

Lemma 1. Assume that $u$ in $L^{\infty}\left([0,1], L^{2}\left(\mathbb{R}^{n}\right)\right) \cap L^{2}\left([0,1], H^{1}\left(\mathbb{R}^{n}\right)\right)$ satisfies

$$
\partial_{t} u=(A+i B)(\Delta u+V(x, t) u+F(x, t)) \quad \text { in } \mathbb{R}^{n} \times(0,1],
$$

$A>0$ and $B \in \mathbb{R}$. Then

$$
\begin{aligned}
& e^{-M_{T}}\left\|e^{\frac{\gamma A|x|^{2}}{A+4 \gamma\left(A^{2}+B^{2}\right) T}} u(T)\right\| \\
& \leq\left\|e^{\gamma|x|^{2}} u(0)\right\|+\sqrt{A^{2}+B^{2}}\left\|e^{\frac{\gamma A|x|^{2}}{A+4 \gamma\left(A^{2}+B^{2}\right) t}} F(t)\right\|_{L^{1}\left([0, T], L^{2}\left(\mathbb{R}^{n}\right)\right)}
\end{aligned}
$$

when $\gamma \geq 0,0 \leq T \leq 1$ and $M_{T}=\left\|A(\operatorname{Re} V)^{+}-B \operatorname{Im} V\right\|_{L^{1}\left([0, T], L^{\infty}\left(\mathbb{R}^{n}\right)\right)}$. 
Proof. Write $v=e^{\varphi} u$, where $\varphi$ is a real-valued function to be chosen later. The function $v$ satisfies

$$
\partial_{t} v=\mathcal{S} v+\mathcal{A} v+(A+i B) e^{\varphi} F \quad \text { in } \mathbb{R}^{n} \times(0,1],
$$

where the symmetric and skew-symmetric operators $\mathcal{S}$ and $\mathcal{A}$ are given by

$$
\begin{aligned}
& \mathcal{S}=A\left(\Delta+|\nabla \varphi|^{2}\right)-i B(2 \nabla \varphi \cdot \nabla+\Delta \varphi)+\left(\partial_{t} \varphi+A \operatorname{Re} V-B \operatorname{Im} V\right), \\
& \mathcal{A}=i B\left(\Delta+|\nabla \varphi|^{2}\right)-A(2 \nabla \varphi \cdot \nabla+\Delta \varphi)+i(B \operatorname{Re} V+A \operatorname{Im} V) .
\end{aligned}
$$

To prove Lemma 1 we use the energy method and try to keep track of the decay of the $L^{2}\left(\mathbb{R}^{n}\right)$-norm of $v$. Formally,

$$
\partial_{t}\|v\|^{2}=2 \operatorname{Re}(\mathcal{S} v, v)+2 \operatorname{Re}\left((A+i B) e^{\varphi} F, v\right)
$$

when $t \geq 0$. Again, a formal integration by parts gives

$$
\begin{aligned}
\operatorname{Re}(\mathcal{S} v, v)= & -A \int_{\mathbb{R}^{n}}|\nabla v|^{2} d x+\int_{\mathbb{R}^{n}}\left(A|\nabla \varphi|^{2}+\partial_{t} \varphi\right)|v|^{2} d x \\
& +2 B \operatorname{Im} \int_{\mathbb{R}^{n}} \bar{v} \nabla \varphi \cdot \nabla v d x+\int_{\mathbb{R}^{n}}(A \operatorname{Re} V-B \operatorname{Im} V)|v|^{2} d x
\end{aligned}
$$

and the Cauchy-Schwarz inequality implies that

$$
\partial_{t}\|v(t)\|^{2} \leq 2\left\|A(\operatorname{Re} V(t))^{+}-B \operatorname{Im} V(t)\right\|_{\infty}\|v(t)\|^{2}+2 \sqrt{A^{2}+B^{2}}\left\|e^{\varphi} F(t)\right\|\|v(t)\|
$$

when

$$
\left(A+B^{2} / A\right)|\nabla \varphi|^{2}+\partial_{t} \varphi \leq 0 \quad \text { in } \mathbb{R}_{+}^{n+1} .
$$

When $\varphi(x, t)=a(t) \phi(x)$, it suffices that

$$
a(t)^{2}\left(A+B^{2} / A\right)|\nabla \phi(x)|^{2}+a^{\prime}(t) \phi(x) \leq 0 .
$$

At the end we shall require that $\phi(x)=|x|^{2}$. In that case the latter holds when

$$
\left\{\begin{array}{l}
a^{\prime}(t)=-4\left(A+B^{2} / A\right) a(t)^{2}, \\
a(0)=\gamma .
\end{array}\right.
$$

To formalize the integration by parts and calculations carried out above, given $\gamma>0$, we truncate $|x|^{2}$ as

$$
\phi_{R}(x)= \begin{cases}|x|^{2}, & |x| \leq R, \\ R^{2}, & |x|>R,\end{cases}
$$

regularize $\phi_{R}$ with a radial mollifier $\theta_{\rho}$ and set

$$
\varphi_{\rho, R}(x, t)=a(t) \theta_{\rho} * \phi_{R}(x), \quad v_{\rho, R}=e^{\varphi_{\rho, R}} u,
$$

where

$$
a(t)=\frac{\gamma A}{A+4 \gamma\left(A^{2}+B^{2}\right) t}
$$

is the solution to 2.3 . 
Because the right hand side of 2.1) only involves the first derivatives of $\varphi, \phi_{R}$ is Lipschitz and bounded at infinity,

$$
\theta_{\rho} * \phi_{R} \leq \theta_{\rho} *|x|^{2}=|x|^{2}+C(n) \rho^{2},
$$

and 2.2 holds uniformly in the variables $\rho$ and $R$, when $\varphi$ is replaced by $\varphi_{\rho, R}$, it follows (and now rigorously) that the estimate

$$
\left\|v_{\rho, R}(T)\right\| \leq e^{M_{T}}\left(\left\|e^{\gamma|x|^{2}} u(0)\right\|+\sqrt{A^{2}+B^{2}}\left\|e^{\varphi_{\rho, R}} F\right\|_{L^{1}\left([0, T], L^{2}\left(\mathbb{R}^{n}\right)\right)}\right)
$$

holds uniformly in $\rho$ and $R$. Lemma 1 follows after letting $\rho$ tend to zero and $R$ to infinity.

Lemma 2. Suppose that $\mathcal{S}$ is a symmetric operator, $\mathcal{A}$ is skew-symmetric, both are allowed to depend on the time variable, $G$ is a positive function, $f(x, t)$ is a reasonable function,

$$
H(t)=(f, f), \quad D(t)=(\mathcal{S} f, f), \quad \partial_{t} \mathcal{S}=\mathcal{S}_{t}, \quad N(t)=\frac{D(t)}{H(t)} .
$$

Then

$$
\begin{gathered}
\partial_{t}^{2} H=2 \partial_{t} \operatorname{Re}\left(\partial_{t} f-\mathcal{S} f-\mathcal{A} f, f\right)+2\left(\mathcal{S}_{t} f+[\mathcal{S}, \mathcal{A}] f, f\right) \\
+\left\|\partial_{t} f-\mathcal{A} f+\mathcal{S} f\right\|^{2}-\left\|\partial_{t} f-\mathcal{A} f-\mathcal{S} f\right\|^{2}
\end{gathered}
$$

and

$$
\dot{N}(t) \geq\left(\mathcal{S}_{t} f+[\mathcal{S}, \mathcal{A}] f, f\right) / H-\left\|\partial_{t} f-\mathcal{A} f-\mathcal{S} f\right\|^{2} / 2 H .
$$

Moreover, if

$$
\left|\partial_{t} f-\mathcal{A} f-\mathcal{S} f\right| \leq M_{1}|f|+G \quad \text { in } \mathbb{R}^{n} \times[0,1], \quad \mathcal{S}_{t}+[\mathcal{S}, \mathcal{A}] \geq-M_{0},
$$

and

$$
M_{2}=\sup _{[0,1]}\|G(t)\| /\|f(t)\|
$$

is finite, then $\log H(t)$ is "logarithmically convex" in $[0,1]$ and there is a universal constant $N$ such that

$$
H(t) \leq e^{N\left(M_{0}+M_{1}+M_{2}+M_{1}^{2}+M_{2}^{2}\right)} H(0)^{1-t} H(1)^{t} \quad \text { when } 0 \leq t \leq 1 .
$$

Proof. Formally,

$$
\dot{H}(t)=2 \operatorname{Re}\left(\partial_{t} f, f\right)=2 \operatorname{Re}\left(\partial_{t} f-\mathcal{S} f-\mathcal{A} f, f\right)+2(\mathcal{S} f, f)
$$

and

$$
\dot{H}(t)=2 \operatorname{Re}\left(\partial_{t} f-\mathcal{S} f-\mathcal{A} f, f\right)+2 D(t) .
$$

Also,

$$
\begin{aligned}
& \dot{H}(t)=\operatorname{Re}\left(\partial_{t} f+\mathcal{S} f, f\right)+\operatorname{Re}\left(\partial_{t} f-\mathcal{S} f, f\right), \\
& D(t)=\frac{1}{2} \operatorname{Re}\left(\partial_{t} f+\mathcal{S} f, f\right)-\frac{1}{2} \operatorname{Re}\left(\partial_{t} f-\mathcal{S} f, f\right),
\end{aligned}
$$


and multiplying the last two formulae gives

$$
\dot{H}(t) D(t)=\frac{1}{2}\left(\operatorname{Re}\left(\partial_{t} f+\mathcal{S} f, f\right)\right)^{2}-\frac{1}{2}\left(\operatorname{Re}\left(\partial_{t} f-\mathcal{S} f, f\right)\right)^{2} .
$$

Adding a skew-symmetric operator does not change the real parts, and so

$$
\dot{H}(t) D(t)=\frac{1}{2}\left(\operatorname{Re}\left(\partial_{t} f-\mathcal{A} f+\mathcal{S} f, f\right)\right)^{2}-\frac{1}{2}\left(\operatorname{Re}\left(\partial_{t} f-\mathcal{A} f-\mathcal{S} f, f\right)\right)^{2} .
$$

Differentiating $D(t)$, we obtain

$$
\begin{aligned}
\dot{D}(t) & =\left(\mathcal{S}_{t} f, f\right)+\left(\mathcal{S} \partial_{t} f, f\right)+\left(\mathcal{S} f, \partial_{t} f\right)=\left(\mathcal{S}_{t} f, f\right)+2 \operatorname{Re}\left(\partial_{t} f, \mathcal{S} f\right) \\
& =\left(\mathcal{S}_{t} f+[\mathcal{S}, \mathcal{A}] f, f\right)+2 \operatorname{Re}\left(\partial_{t} f-\mathcal{A} f, \mathcal{S} f\right)
\end{aligned}
$$

and the polarization identity gives

$$
\dot{D}(t)=\left(\mathcal{S}_{t} f+[\mathcal{S}, \mathcal{A}] f, f\right)+\frac{1}{2}\left\|\partial_{t} f-\mathcal{A} f+\mathcal{S} f\right\|^{2}-\frac{1}{2}\left\|\partial_{t} f-\mathcal{A} f-\mathcal{S} f\right\|^{2} .
$$

The formula (2.4) for the second derivative of $H$ follows from 2.7] and 2.9]. The identity

$$
\begin{aligned}
\dot{N}(t)= & \left(\mathcal{S}_{t} f+[\mathcal{S}, \mathcal{A}] f, f\right) / H \\
& +\frac{1}{2}\left[\left\|\partial_{t} f-\mathcal{A} f+\mathcal{S} f\right\|^{2}\|f\|^{2}-\left(\operatorname{Re}\left(\partial_{t} f-\mathcal{A} f+\mathcal{S} f, f\right)\right)^{2}\right] / H^{2} \\
& +\frac{1}{2}\left[\left(\operatorname{Re}\left(\partial_{t} f-\mathcal{A} f-\mathcal{S} f, f\right)\right)^{2}-\left\|\partial_{t} f-\mathcal{A} f-\mathcal{S} f\right\|^{2}\|f\|^{2}\right] / H^{2}
\end{aligned}
$$

follows from 2.8 and 2.9). The first inequality in Lemma 2 follows from the positiveness of the second line (Cauchy-Schwarz inequality) and of the first term on the last line of the previous identity.

When 2.5 holds, the first part in Lemma 2 shows that

$$
\dot{N}(t) \geq-\left(M_{0}+M_{1}^{2}+M_{2}^{2}\right),
$$

and from 2.7 ,

$$
\partial_{t}(\log H(t)+\mathrm{O}(1))=2 N(t) .
$$

Altogether,

$$
\partial_{t}^{2}(\log H(t)+\mathrm{O}(1)) \geq 0 \quad \text { when } 0 \leq t \leq 1
$$

where $\mathrm{O}(1)$ is a function satisfying $|\mathrm{O}(1)| \leq N\left(M_{0}+M_{1}+M_{2}+M_{1}^{2}+M_{2}^{2}\right)$ in $[0,1]$. The integration of the inequality

$$
\partial_{s}(\log H(s)+\mathrm{O}(1)) \leq \partial_{\tau}(\log H(\tau)+\mathrm{O}(1)) \quad \text { when } 0 \leq s \leq t \leq \tau \leq 1
$$

over the intervals $0 \leq s \leq t$ and $t \leq \tau \leq 1$ implies 2.6. 
Lemma 3. Assume that $u$ in $L^{\infty}\left([0,1], L^{2}\left(\mathbb{R}^{n}\right)\right) \cap L^{2}\left([0,1], H^{1}\left(\mathbb{R}^{n}\right)\right)$ satisfies

$$
\partial_{t} u=(A+i B)(\Delta u+V(x, t) u+F(x, t)) \quad \text { in } \mathbb{R}^{n} \times[0,1],
$$

where $A>0, B \in \mathbb{R}, V$ is complex-valued, $\gamma>0$, and $\sup _{[0,1]}\|V(t)\|_{\infty} \leq M_{1}$. Set

$$
M_{2}=\sup _{[0,1]}\left\|e^{\gamma|x|^{2}} F(t)\right\| /\|u(t)\|
$$

and assume that $\left\|e^{\gamma|x|^{2}} u(0)\right\|,\left\|e^{\gamma|x|^{2}} u(1)\right\|$ and $M_{2}$ are finite. Then $\left\|e^{\gamma|x|^{2}} u(t)\right\|$ is "logarithmically convex" in $[0,1]$ and there is a universal constant $N$ such that

$$
\begin{aligned}
& \left\|e^{\gamma|x|^{2}} u(t)\right\| \\
& \quad \leq e^{N\left[\left(A^{2}+B^{2}\right)\left(\gamma M_{1}^{2}+M_{2}^{2}\right)+\sqrt{A^{2}+B^{2}}\left(M_{1}+M_{2}\right)\right]}\left\|e^{\gamma|x|^{2}} u(0)\right\|^{1-t}\left\|e^{\gamma|x|^{2}} u(1)\right\|^{t}
\end{aligned}
$$

when $0 \leq t \leq 1$.

Proof. Let $f=e^{\gamma \varphi} u$, where $\varphi=\varphi(x, t)$ is to be chosen. The function $f$ satisfies

$$
\partial_{t} f=\delta f+\mathcal{A} f+(A+i B)\left(V f+e^{\gamma \varphi} F\right) \quad \text { in } \mathbb{R}_{+}^{n+1},
$$

with symmetric and skew-symmetric operators $\mathcal{S}$ and $\mathcal{A}$,

$$
\begin{aligned}
\mathcal{S} & =A\left(\Delta+\gamma^{2}|\nabla \varphi|^{2}\right)-i B \gamma(2 \nabla \varphi \cdot \nabla+\Delta \varphi)+\gamma \partial_{t} \varphi, \\
\mathcal{A} & =i B\left(\Delta+\gamma^{2}|\nabla \varphi|^{2}\right)-A \gamma(2 \nabla \varphi \cdot \nabla+\Delta \varphi) .
\end{aligned}
$$

A calculation shows that

$$
\begin{aligned}
\mathcal{S}_{t}+[\mathcal{S}, \mathcal{A}]= & \gamma \partial_{t}^{2} \varphi+4 \gamma^{2} A \nabla \varphi \cdot \nabla \partial_{t} \varphi-2 i B \gamma\left(2 \nabla \partial_{t} \varphi \cdot \nabla+\Delta \partial_{t} \varphi\right) \\
& -\gamma\left(A^{2}+B^{2}\right)\left[4 \nabla \cdot\left(D^{2} \varphi \nabla\right)-4 \gamma^{2} D^{2} \varphi \nabla \varphi \cdot \nabla \varphi+\Delta^{2} \varphi\right] .
\end{aligned}
$$

At the end we shall require that $\varphi(x, t)=|x|^{2}$; then

$$
\mathcal{S}_{t}+[\mathcal{S}, \mathcal{A}]=-\gamma\left(A^{2}+B^{2}\right)\left[8 \Delta-32 \gamma^{2}|x|^{2}\right]
$$

and

$$
\left(\mathcal{S}_{t} f+[\mathcal{S}, \mathcal{A}] f, f\right)=\gamma\left(A^{2}+B^{2}\right) \int_{\mathbb{R}^{n}}\left(8|\nabla f|^{2}+32 \gamma^{2}|x|^{2}|f|^{2}\right) d x .
$$

This identity, the boundedness of $V$, and (2.12) imply that

$$
\left|\partial_{t} f-\mathcal{S} f-\mathcal{A} f\right| \leq \sqrt{A^{2}+B^{2}}\left(M_{1}|f|+e^{\gamma \varphi}|F|\right), \quad \mathcal{S}_{t}+[\mathcal{S}, \mathcal{A}] \geq 0,
$$

and if we knew that the quantities and calculations involved in the proof of Lemma 2 were finite and correct when $f=e^{\gamma|x|^{2}} u$, we would have the "logarithmic convexity" of $H(t)=\left\|e^{\gamma|x|^{2}} u(t)\right\|^{2}$ and get 2.11) from Lemma2. 
To justify the validity of the previous arguments, given $a$ and $\rho$ in $(0,1)$, define

$$
\varphi_{a}(x)= \begin{cases}|x|^{2}, & |x|<1, \\ \left(2|x|^{2-a}-a\right) /(2-a), & |x| \geq 1,\end{cases}
$$

and replace $\varphi=|x|^{2}$ by $\varphi_{a, \rho}=\theta_{\rho} * \varphi_{a}$, where $\theta$ in $C_{0}^{\infty}\left(\mathbb{R}^{n}\right)$ is a radial function. Observe that $\varphi_{a}$ is a $C^{1,1}\left(\mathbb{R}^{n}\right)$ convex function, $\varphi_{a, \rho} \leq|x|^{2}+C(n) \rho^{2}, \varphi_{a, \rho}$ is convex and grows at infinity not faster than $|x|^{2-a}$. At the same time,

$$
\Delta \varphi_{a}(x)= \begin{cases}2 n, & |x| \leq 1 \\ 2(n-a)|x|^{-a}, & |x| \geq 1\end{cases}
$$

and the distribution $\partial_{j} \Delta \varphi_{a}, j=1, \ldots, n$, is equal to

$$
-2 a x_{j} d \sigma-2 a(n-a) x_{j}|x|^{-a-2} \chi_{\mathbb{R}^{n} \backslash B_{1}},
$$

where $d \sigma$ is the surface measure on $\partial B_{1}$. This and the identity

$$
\Delta^{2} \varphi_{a, \rho}=\sum_{j=1}^{n} \partial_{j} \theta_{\rho} * \partial_{j} \Delta \varphi_{a}
$$

show that

$$
\left\|\Delta^{2} \varphi_{a, \rho}\right\|_{\infty} \leq C(n, \rho) a .
$$

Set then $f_{a, \rho}=e^{\gamma \varphi_{a, \rho}} u$ and $H_{a, \rho}(t)=\left\|f_{a, \rho}\right\|^{2}$ in Lemma 2. The decay bound in Lemma 1 and the interior regularity for solutions of 2.10 (here we use that $A$ is positive) can now be used qualitatively to make sure that the quantities or calculations involved in the proof of Lemma 2 are finite and correct for $f_{a, \rho}$. In this case, $f_{a, \rho}$ satisfies

$$
\partial_{t} f_{a, \rho}=\mathcal{S}^{a, \rho} f_{a, \rho}+\mathcal{A}^{a, \rho} f_{a, \rho}+(A+i B)\left(V f_{a, \rho}+e^{\gamma \varphi_{a, \rho}} F\right) \quad \text { in } \mathbb{R}^{n} \times[0,1],
$$

with symmetric and skew-symmetric operators $\mathcal{S}^{a, \rho}$ and $\mathcal{A}^{a, \rho}$ given by 2.13 with $\varphi$ replaced by $\varphi_{a, \rho}$. The formula for the operator

$$
\mathcal{S}_{t}^{a, \rho}+\left[\mathcal{S}^{a, \rho}, \mathcal{A}^{a, \rho}\right]
$$

in (2.14), the convexity of $\varphi_{a, \rho}$, and the bounds (2.17) and (2.18) imply the inequalities

$$
\begin{aligned}
\left|\partial_{t} f_{a, \rho}-\mathcal{S}^{a, \rho} f_{a, \rho}-\mathcal{A}^{a, \rho} f_{a, \rho}\right| & \leq \sqrt{A^{2}+B^{2}}\left(M_{1}\left|f_{a, \rho}\right|+e^{\gamma \varphi_{a, \rho}} F\right), \\
S_{t}^{a, \rho}+\left[\mathcal{S}^{a, \rho}, \mathcal{A}^{a, \rho}\right] & \geq 0,
\end{aligned}
$$

and $M_{2}(a, \rho) \leq e^{C(n) \rho^{2}} M_{2}$ when $0<a, \rho<1$. In particular, $H_{a, \rho}$ is "logarithmically convex" in $[0,1]$ and

$$
H_{a, \rho}(t) \leq e^{N\left[\left(A^{2}+B^{2}\right)\left(M_{1}^{2}+M_{2}^{2}\right)+\sqrt{A^{2}+B^{2}}\left(M_{1}+M_{2}\right)\right]} H_{a, \rho}(0)^{1-t} H_{a, \rho}(1)^{t} .
$$

Then (2.11) follows after taking first the limit when $a$ tends to zero in 2.19), and then when $\rho$ tends to zero. 
Lemma 4. Assume that $A+i B, u$, and $V$ are as in Lemma 3 and $\gamma>0$. Then

$$
\begin{aligned}
& \left\|\sqrt{t(1-t)} e^{\gamma|x|^{2}} \nabla u\right\|_{L^{2}\left(\mathbb{R}^{n} \times[0,1]\right)}+\left\|\sqrt{t(1-t)}|x| e^{\gamma|x|^{2}} u\right\|_{L^{2}\left(\mathbb{R}^{n} \times[0,1]\right)} \\
& \leq N\left[\left(1+M_{1}\right) \sup _{[0,1]}\left\|e^{\gamma|x|^{2}} u(t)\right\|+\sup _{[0,1]}\left\|e^{\gamma|x|^{2}} F\right\|_{L^{2}\left(\mathbb{R}^{n} \times[0,1]\right)}\right],
\end{aligned}
$$

where $N$ remains bounded when $\gamma$ and $A^{2}+B^{2}$ are bounded below.

Proof. A formal integration by parts shows that

$$
\int_{\mathbb{R}^{n}}\left(|\nabla f|^{2}+4 \gamma^{2}|x|^{2}|f|^{2}\right) d x=\int_{\mathbb{R}^{n}} e^{2 \gamma|x|^{2}}\left(|\nabla u|^{2}-2 n \gamma|u|^{2}\right) d x
$$

when $f=e^{\gamma|x|^{2}} u$, while either well known properties of Hermite functions [17] or integration by parts, the Cauchy-Schwarz inequality, and the identity $n=\nabla \cdot x$ give

$$
\int_{\mathbb{R}^{n}}\left(|\nabla f|^{2}+4 \gamma^{2}|x|^{2}|f|^{2}\right) d x \geq 2 \gamma n \int_{\mathbb{R}^{n}}|f|^{2} d x .
$$

The sum of the last two formulae gives the inequality

$$
2 \int_{\mathbb{R}^{n}}\left(|\nabla f|^{2}+4 \gamma^{2}|x|^{2}|f|^{2}\right) d x \geq \int_{\mathbb{R}^{n}} e^{2 \gamma|x|^{2}}|\nabla u|^{2} d x
$$

Integration over [0, 1] of $t(1-t)$ times the formula 2.4 for the second derivative of $H(t)=\|f(t)\|^{2}$ and integration by parts show that in the general framework of Lemma 2 .

$$
\begin{aligned}
2 \int_{0}^{1} t(1-t)\left(\mathcal{S}_{t} f+[\mathcal{S}, \mathcal{A}] f, f\right) d t+2 \int_{0}^{1} H(t) d t \\
\leq H(1)+H(0)+2 \int_{0}^{1}(1-2 t) \operatorname{Re}\left(\partial_{t} f-\mathcal{S} f-\mathcal{A} f, f\right) d t \\
\quad+\int_{0}^{1} t(1-t)\left\|\partial_{t} f-\mathcal{A} f-\mathcal{S} f\right\|^{2} d t
\end{aligned}
$$

Assuming again that the last two calculations are justified for $f=e^{\gamma|x|^{2}} u$, 2.22, 2.15), 2.16), 2.21) and the identity $\nabla f=e^{\gamma|x|^{2}}(\nabla u+2 \gamma x u)$ imply the lemma.

The interior regularity of the solutions to 2.10 (here we use again that $A>0$ ) shows that the calculations leading to 2.21) and 2.22 are justified when $f=e^{(\gamma-\rho)|x|^{2}} u$, $0<\rho<\gamma$, and the right-hand side of 2.20$]$ is finite. The lemma follows on letting $\rho$ tend to zero. 


\section{The conformal or Appell transformation}

Lemma 5. Assume that $u(y, s)$ satisfies

$$
\partial_{s} u=(A+i B)(\Delta u+V(y, s) u+F(y, s)) \quad \text { in } \mathbb{R}^{n} \times[0,1],
$$

$A+i B \neq 0, \alpha$ and $\beta$ are positive, $\gamma \in \mathbb{R}$, and set

$$
\tilde{u}(x, t)=\left(\frac{\sqrt{\alpha \beta}}{\alpha(1-t)+\beta t}\right)^{n / 2} u\left(\frac{\sqrt{\alpha \beta} x}{\alpha(1-t)+\beta t}, \frac{\beta t}{\alpha(1-t)+\beta t}\right) e^{\frac{(\alpha-\beta)|x|^{2}}{4(A+i B)(\alpha(1-t)+\beta t)}} .
$$

Then $\tilde{u}$ satisfies

$$
\partial_{t} \tilde{u}=(A+i B)(\Delta \tilde{u}+\widetilde{V}(x, t) \tilde{u}+\widetilde{F}(x, t)) \quad \text { in } \mathbb{R}^{n} \times[0,1],
$$

with

$$
\begin{aligned}
& \tilde{V}(x, t)=\frac{\alpha \beta}{(\alpha(1-t)+\beta t)^{2}} V\left(\frac{\sqrt{\alpha \beta} x}{\alpha(1-t)+\beta t}, \frac{\beta t}{\alpha(1-t)+\beta t}\right), \\
& \widetilde{F}(x, t)=\left(\frac{\sqrt{\alpha \beta}}{\alpha(1-t)+\beta t}\right)^{n / 2+2} F\left(\frac{\sqrt{\alpha \beta} x}{\alpha(1-t)+\beta t}, \frac{\beta t}{\alpha(1-t)+\beta t}\right) e^{\frac{(\alpha-\beta)|x|^{2}}{4(A+i B)(\alpha(1-t)+\beta t)}} .
\end{aligned}
$$

Moreover,

$$
\left\|e^{\gamma|x|^{2}} \tilde{F}(t)\right\|=\frac{\alpha \beta}{(\alpha(1-t)+\beta t)^{2}}\left\|e^{\left[\frac{\gamma \alpha \beta}{(\alpha s+\beta(1-s))^{2}}+\frac{(\alpha-\beta) A}{4\left(A^{2}+B^{2}\right)(\alpha s+\beta(1-s))}\right]|y|^{2}} F(s)\right\|
$$

and

$$
\left\|e^{\gamma|x|^{2}} \tilde{u}(t)\right\|=\left\|e^{\left[\frac{\gamma \alpha \beta}{(\alpha s+\beta(1-s))^{2}}+\frac{(\alpha-\beta) A}{4\left(A^{2}+B^{2}\right)(\alpha s+\beta(1-s))}\right]|y|^{2}} u(s)\right\|
$$

when $s=\frac{\beta t}{\alpha(1-t)+\beta t}$ and $\gamma \in \mathbb{R}$.

Proof. When $u$ satisfies

$$
\partial_{s} u=(A+i B)(\Delta u+H(y, s)) \quad \text { in } \mathbb{R}^{n} \times[0,1],
$$

the function $u_{1}(x, t)=u(\sqrt{r} x, r t+\tau)$ satisfies

$$
\partial_{t} u_{1}=(A+i B)\left(\Delta u_{1}+r H(\sqrt{r} x, r t+\tau)\right),
$$

and $u_{2}(x, t)=t^{-n / 2} u(x / t, 1 / t) e^{\frac{|x|^{2}}{4(A+i B) t}}$ is a solution to

$$
\partial_{t} u_{2}=-(A+i B)\left(\Delta u_{2}+t^{-n / 2-2} H(x / t, 1 / t) e^{\frac{|x|^{2}}{4(A+i B) t}}\right) .
$$

These two facts and the sequence of changes of variables below prove the lemma when $\alpha>\beta$ :

$$
u\left(\sqrt{\frac{\alpha \beta}{\alpha-\beta}} x, \frac{\alpha \beta}{\alpha-\beta} t-\frac{\beta}{\alpha-\beta}\right)
$$


is a solution to the same non-homogeneous equation but with right-hand side

$$
\frac{\alpha \beta}{\alpha-\beta} H\left(\sqrt{\frac{\alpha \beta}{\alpha-\beta}} x, \frac{\alpha \beta}{\alpha-\beta} t-\frac{\beta}{\alpha-\beta}\right) .
$$

The function

$$
\frac{1}{(\alpha-t)^{n / 2}} u\left(\frac{\sqrt{\alpha \beta} x}{\sqrt{\alpha-\beta}(\alpha-t)}, \frac{\alpha \beta}{(\alpha-\beta)(\alpha-t)}-\frac{\beta}{\alpha-\beta}\right) e^{\frac{|x|^{2}}{4(A+i B)(\alpha-t)}}
$$

satisfies 3.1) with right-hand side

$$
\frac{\alpha \beta}{(\alpha-\beta)(\alpha-t)^{n / 2+2}} H\left(\frac{\sqrt{\alpha \beta} x}{\sqrt{\alpha-\beta}(\alpha-t)}, \frac{\alpha \beta}{(\alpha-\beta)(\alpha-t)}-\frac{\beta}{\alpha-\beta}\right) e^{\frac{|x|^{2}}{4(A+i B)(\alpha-t)}} .
$$

Replacing $(x, t)$ by $(\sqrt{\alpha-\beta} x,(\alpha-\beta) t)$, we find that

$$
\frac{1}{(\alpha(1-t)+\beta t)^{n / 2}} u\left(\frac{\sqrt{\alpha \beta} x}{\alpha(1-t)+\beta t}, \frac{\alpha \beta}{(\alpha-\beta)(\alpha(1-t)+\beta t)}-\frac{\beta}{\alpha-\beta}\right) e^{\frac{(\alpha-\beta)|x|^{2}}{4(A+i B)(\alpha(1-t)+\beta t)}}
$$

is a solution to 3.1 but with right-hand side

$\frac{\alpha \beta}{(\alpha+\beta-\alpha t)^{n / 2+2}} H\left(\frac{\sqrt{\alpha \beta} x}{\alpha(1-t)+\beta t}, \frac{\alpha \beta}{(\alpha-\beta)(\alpha(1-t)+\beta t)}-\frac{\beta}{\alpha-\beta}\right) e^{\frac{(\alpha-\beta)|x|^{2}}{4(A+i B)(\alpha(1-t)+\beta t)}}$.

Finally, observe that

$$
s=\frac{\beta t}{\alpha(1-t)+\beta t}=\frac{\alpha \beta}{(\alpha-\beta)(\alpha(1-t)+\beta t)}-\frac{\beta}{\alpha-\beta}
$$

and multiply 3.2 and 3.3 by $(\sqrt{\alpha \beta})^{n / 2}$.

The case $\beta>\alpha$ follows by reversing the time with the changes of variables $s^{\prime}=1-s$ and $t^{\prime}=1-t$. The relations between the different norms of $\widetilde{u}, u, \widetilde{F}$, and $F$ follow by undoing the changes of variables and using the identity

$$
\frac{\sqrt{\alpha \beta}}{\alpha(1-t)+\beta t}=\frac{\alpha s+\beta(1-s)}{\sqrt{\alpha \beta}} .
$$

\section{Variable coefficients. Proof of Theorem 3}

Proof of Theorem 3 We may assume that $\alpha \neq \beta$. The case $\alpha=\beta$ follows from the former by replacing $\beta$ by $\beta+\delta, \delta>0$, and letting $\delta$ tend to zero. We may also assume that $\alpha<\beta$. Otherwise, replace $u$ by $\bar{u}(1-t)$. Set then $H=\Delta+V_{1}(x)$ and let $e^{t(A+i B) H} u_{0}$ denote the $C\left([0,1], L^{2}\left(\mathbb{R}^{n}\right)\right)$ solution to

$$
\left\{\begin{array}{l}
\partial_{t} v=(A+i B)\left(\Delta v+V_{1}(x) v\right) \quad \text { in } \mathbb{R}^{n} \times[0,1], \\
v(0)=u_{0}
\end{array}\right.
$$


when $\operatorname{Re}(A+i B) \geq 0$. The Duhamel principle shows that

$$
u(t)=e^{i t H} u(0)+i \int_{0}^{t} e^{i(t-s) H}\left(V_{2}(s) u(s)\right) d s \quad \text { in } \mathbb{R}^{n} \times[0,1] .
$$

For $0 \leq \epsilon \leq 1$, set

$$
\begin{aligned}
& F_{\epsilon}(t)=\frac{i}{\epsilon+i} e^{\epsilon t H}\left(V_{2}(t) u(t)\right), \\
& u_{\epsilon}(t)=e^{(\epsilon+i) t H} u(0)+(\epsilon+i) \int_{0}^{t} e^{(\epsilon+i)(t-s) H} F_{\epsilon}(s) d s .
\end{aligned}
$$

Then $u_{\epsilon}$ is in $L^{\infty}\left([0,1], L^{2}\left(\mathbb{R}^{n}\right)\right) \cap L^{2}\left([0,1], H^{1}\left(\mathbb{R}^{n}\right)\right)$ and satisfies

$$
\left\{\begin{array}{l}
\partial_{t} u_{\epsilon}=(\epsilon+i)\left(H u_{\epsilon}+F_{\epsilon}(t)\right) \quad \text { in } \mathbb{R}^{n} \times[0,1], \\
u_{\epsilon}(0)=u(0) .
\end{array}\right.
$$

The identities [14]

$$
e^{\left(z_{1}+z_{2}\right) H}=e^{\left(z_{2}+z_{1}\right) H}=e^{z_{1} H} e^{z_{2} H} \quad \text { when } \operatorname{Re} z_{1}, \operatorname{Re} z_{2} \geq 0,
$$

4.1, 4.2 and 4.3) show that

$$
u_{\epsilon}(t)=e^{\epsilon t H} u(t) \quad \text { when } 0 \leq t \leq 1 .
$$

In particular,

$$
u_{\epsilon}(1)=e^{\epsilon H} u(1),
$$

and Lemma 1 with $A+i B=\epsilon, \gamma=1 / \beta^{2}, F \equiv 0$ and the fact that $u_{\epsilon}(0)=u(0)$ imply that

$$
\left\|e^{\frac{|x|^{2}}{\beta^{2}+4 \epsilon}} u_{\epsilon}(1)\right\| \leq e^{\epsilon\left\|V_{1}\right\|_{L^{\infty}\left(\mathbb{R}^{n}\right)}}\left\|e^{|x|^{2} / \beta^{2}} u(1)\right\|, \quad\left\|e^{|x|^{2} / \alpha^{2}} u_{\epsilon}(0)\right\|=\left\|e^{|x|^{2} / \alpha^{2}} u(0)\right\| .
$$

A second application of Lemma 1 with $A+i B=\epsilon, F \equiv 0, \gamma=1 /(\alpha t+\beta(1-t))^{2}$ and (4.2) show that

$$
\left\|\epsilon^{\frac{|x|^{2}}{(\alpha t+\beta(1-t))^{2}+4 \epsilon t}} F_{\epsilon}(t)\right\| \leq e^{\epsilon\left\|V_{1}\right\|_{\infty}}\left\|\epsilon^{\frac{|x|^{2}}{(\alpha t+\beta(1-t))^{2}}} V_{2}(t)\right\|_{\infty}\|u(t)\|
$$

when $0 \leq t \leq 1$. If we set $\alpha_{\epsilon}=\alpha+2 \epsilon$ and $\beta_{\epsilon}=\beta+2 \epsilon$, the last three inequalities give

$$
\begin{gathered}
\left\|e^{|x|^{2} / \beta_{e}^{2}} u_{\epsilon}(1)\right\| \leq e^{\epsilon\left\|V_{1}\right\|_{\infty}}\left\|e^{|x|^{2} / \beta^{2}} u(1)\right\|, \quad\left\|e^{|x|^{2} / \alpha_{\epsilon}^{2}} u_{\epsilon}(0)\right\| \leq\left\|e^{|x|^{2} / \alpha^{2}} u(0)\right\|, \\
\left\|e^{\frac{|x|^{2}}{\left(\alpha_{\epsilon} t+\beta_{\epsilon}(1-t)\right)^{2}}} F_{\epsilon}(t)\right\| \leq e^{\epsilon\left\|V_{1}\right\| \infty}\left\|e^{\frac{|x|^{2}}{(\alpha t+\beta(1-t))^{2}}} V_{2}(t)\right\|_{\infty}\|u(t)\| .
\end{gathered}
$$

A third application of Lemma 1 with $A+i B=\epsilon, F \equiv 0, \gamma=0$, and 4.2, 4.5 imply that

$$
\left\|F_{\epsilon}(t)\right\| \leq e^{\epsilon\left\|V_{1}\right\|_{\infty}}\left\|V_{2}(t)\right\|_{L^{\infty}\left(\mathbb{R}^{n}\right)}\|u(t)\|, \quad\left\|u_{\epsilon}(t)\right\| \leq e^{\epsilon\left\|V_{1}\right\| \infty}\|u(t)\|
$$


when $0 \leq t \leq 1$. Set then $\gamma_{\epsilon}=1 / \alpha_{\epsilon} \beta_{\epsilon}$ and let

$\tilde{u}_{\epsilon}(x, t)$

$$
=\left(\frac{\sqrt{\alpha_{\epsilon} \beta_{\epsilon}}}{\alpha_{\epsilon}(1-t)+\beta_{\epsilon} t}\right)^{n / 2} u_{\epsilon}\left(\frac{\sqrt{\alpha_{\epsilon} \beta_{\epsilon}} x}{\alpha_{\epsilon}(1-t)+\beta_{\epsilon} t}, \frac{\beta_{\epsilon} t}{\alpha_{\epsilon}(1-t)+\beta_{\epsilon} t}\right) e^{\frac{\left(\alpha_{\epsilon}-\beta_{\epsilon}|x|^{2}\right.}{4(\epsilon+i)\left(\alpha_{\epsilon}(1-t)+\beta_{\epsilon} t\right)}}
$$

be the function associated to $u_{\epsilon}$ in Lemma 5 when $A+i B=\epsilon+i$ and $\alpha, \beta$ are replaced respectively by $\alpha_{\epsilon}$ and $\beta_{\epsilon}$. Because $\alpha<\beta, \tilde{u}_{\epsilon}$ is in $L^{\infty}\left([0,1], L^{2}\left(\mathbb{R}^{n}\right)\right) \cap$ $L^{2}\left([0,1], H^{1}\left(\mathbb{R}^{n}\right)\right)$ and satisfies

$$
\partial_{t} \tilde{u}_{\epsilon}=(\epsilon+i)\left(\Delta \tilde{u}_{\epsilon}+\tilde{V}_{1}^{\epsilon}(x, t) \tilde{u}_{\epsilon}+\widetilde{F}_{\epsilon}(x, t)\right) \quad \text { in } \mathbb{R}^{n} \times[0,1],
$$

where $\widetilde{V}_{1}^{\epsilon}$ is real-valued,

$$
\begin{gathered}
\widetilde{V}_{1}^{\epsilon}(x, t)=\frac{\alpha_{\epsilon} \beta_{\epsilon}}{\left(\alpha_{\epsilon}(1-t)+\beta_{\epsilon} t\right)^{2}} V_{1}\left(\frac{\sqrt{\alpha_{\epsilon} \beta_{\epsilon}} x}{\alpha_{\epsilon}(1-t)+\beta_{\epsilon} t}\right), \quad \sup _{[0,1]}\left\|\widetilde{V}_{1}^{\epsilon}(t)\right\|_{\infty} \leq \frac{\beta}{\alpha} M_{1}, \\
\widetilde{F}_{\epsilon}(x, t)=\left(\frac{\sqrt{\alpha_{\epsilon} \beta_{\epsilon}}}{\alpha_{\epsilon}(1-t)+\beta_{\epsilon} t}\right)^{n / 2+2} F_{\epsilon}\left(\frac{\sqrt{\alpha_{\epsilon} \beta_{\epsilon}} x}{\alpha_{\epsilon}(1-t)+\beta_{\epsilon} t}, \frac{\beta_{\epsilon} t}{\alpha_{\epsilon}(1-t)+\beta_{\epsilon} t}\right) e^{\frac{\left(\alpha_{\epsilon}-\beta_{\epsilon}|x|^{2}\right.}{4(\epsilon+i)\left(\alpha_{\epsilon}(1-t)+\beta_{\epsilon} t\right)}}, \\
\left\|e^{\gamma_{\epsilon}|x|^{2}} \widetilde{F}_{\epsilon}(t)\right\| \leq \frac{\beta}{\alpha}\left\|\epsilon \frac{|x|^{2}}{\left(\alpha_{\epsilon} t+\beta_{\epsilon}(1-t)\right)^{2}} F_{\epsilon}(s)\right\|, \quad\left\|\widetilde{F}_{\epsilon}(t)\right\| \leq \frac{\beta}{\alpha}\left\|F_{\epsilon}(s)\right\|,
\end{gathered}
$$

and

$$
\begin{gathered}
\left\|e^{\gamma_{\epsilon}|x|^{2}} \tilde{u}_{\epsilon}(t)\right\|=\left\|e^{\left[\frac{1}{\left(\alpha_{\epsilon} s+\beta_{\epsilon}(1-s)\right)^{2}}+\frac{\left(\alpha_{\epsilon}-\beta_{\epsilon}\right) A}{4\left(A^{2}+B^{2}\right)\left(\alpha_{\epsilon} s+\beta_{\epsilon}(1-s)\right)}\right]|y|^{2}} u_{\epsilon}(s)\right\|, \\
\left\|\tilde{u}_{\epsilon}(t)\right\| \leq\left\|u_{\epsilon}(s)\right\|,
\end{gathered}
$$

when $s=\frac{\beta_{\epsilon} t}{\alpha_{\epsilon}(1-t)+\beta_{\epsilon} t}$. The above identity when $t$ is zero or one and (4.6) show that

$$
\left\|e^{\gamma_{\epsilon}|x|^{2}} \tilde{u}_{\epsilon}(0)\right\| \leq\left\|e^{|x|^{2} / \beta^{2}} u(0)\right\|, \quad\left\|e^{\gamma_{\epsilon}|x|^{2}} \widetilde{u}_{\epsilon}(1)\right\| \leq e^{\epsilon\left\|V^{+}\right\| \infty}\left\|e^{|x|^{2} / \beta^{2}} u(1)\right\| .
$$

On the other hand,

$$
N_{1}^{-1}\|u(0)\| \leq\|u(t)\| \leq N_{1}\|u(0)\| \quad \text { when } 0 \leq t \leq 1, \quad N_{1}=e^{\sup _{[0,1]}\left\|\operatorname{Im} V_{2}(t)\right\|_{\infty}},
$$

and the equation satisfied by $\tilde{u}_{\epsilon}$ and the energy method imply that

$$
\partial_{t}\left\|\tilde{u}_{\epsilon}(t)\right\|^{2} \leq 2 \epsilon\left\|\widetilde{V}_{1}^{\epsilon}(t)\right\|_{\infty}\left\|\tilde{u}_{\epsilon}(t)\right\|^{2}+2\left\|\widetilde{F}_{\epsilon}(t)\right\|\left\|\tilde{u}_{\epsilon}(t)\right\| .
$$

Let $0=t_{0}<t_{1}<t_{2}<\cdots<t_{m}=1$ be a uniformly distributed partition of [0,1] where $m$ will be chosen later. The inequality (4.14, , 4.9), the inequality in (4.11), the second inequality in 4.10, 4.8), and 4.13 imply that there is $N_{2}$, which depends on $\beta / \alpha,\left\|V_{1}\right\|_{L^{\infty}\left(\mathbb{R}^{n}\right)}$ and $\sup _{[0,1]}\left\|V_{2}(t)\right\|_{\infty}$, such that

$$
\left\|\widetilde{u}_{\epsilon}\left(t_{i}\right)\right\| \leq e^{(\epsilon \beta / \alpha)\left\|V_{1}\right\|_{\infty}}\left\|\widetilde{u}_{\epsilon}(t)\right\|+N_{2} \sqrt{t_{i}-t_{i-1}}\|u(0)\|
$$


when $t_{i-1} \leq t \leq t_{i}, 0<\epsilon \leq 1$ and $i=1, \ldots, m$. Choose now $m$ so that

$$
N_{2} \max _{1 \leq i \leq m} \sqrt{t_{i}-t_{i-1}} \leq \frac{1}{4 N_{1}}
$$

where $N_{1}$ was defined in 4.13. From $\lim _{\epsilon \rightarrow 0^{+}}\left\|\tilde{u}_{\epsilon}(t)\right\|=\|u(s)\|$ when $s=\frac{\beta t}{\alpha(1-t)+\beta t}$, and 4.13 , we see that there is $\epsilon_{0}$ such that

$$
\left\|\tilde{u}_{\epsilon}\left(t_{i}\right)\right\| \geq \frac{1}{2 N_{1}}\|u(0)\| \quad \text { when } 0<\epsilon \leq \epsilon_{0}, i=1, \ldots, m,
$$

and now 4.17, 4.16 and 4.15) show that

$$
\left\|\tilde{u}_{\epsilon}(t)\right\| \geq \frac{1}{4 N_{1}}\|u(0)\| \quad \text { when } 0<\epsilon \leq \epsilon_{0}, 0 \leq t \leq 1 .
$$

It is now simple to verify that 4.18, the first inequality in 4.10, 4.7), and 4.13) imply that

$$
\sup _{[0,1]} \frac{\left\|e^{\gamma_{\epsilon}|x|^{2}} \widetilde{F}_{\epsilon}(t)\right\|}{\left\|\widetilde{u}_{\epsilon}(t)\right\|} \leq \frac{4 \beta}{\alpha} M_{2}(\epsilon), \quad 0<\epsilon \leq \epsilon_{0},
$$

where

$$
M_{2}(\epsilon)=e^{2 \sup _{[0,1]}\left\|\operatorname{Im} V_{2}(t)\right\|_{\infty}+\epsilon\left\|V_{1}\right\|_{\infty}} \sup _{[0,1]}\left\|e^{\frac{|x|^{2}}{(\alpha t+\beta(1-t))^{2}}} V_{2}(t)\right\|_{\infty}
$$

We can use Lemma 3, 4.12), (4.9) and 4.19) to show that $\left\|e^{\gamma_{\epsilon}|x|^{2}} \widetilde{u}_{\epsilon}(t)\right\|$ is "logarithmically convex" in $[0,1]$ and that

$$
\left\|e^{\gamma_{\epsilon}|x|^{2}} \tilde{u}_{\epsilon}(t)\right\| \leq e^{N\left(M_{1}+M_{2}(\epsilon)+M_{1}^{2}+M_{2}(\epsilon)^{2}\right)}\left\|e^{|x|^{2} / \beta^{2}} u(0)\right\|^{1-t}\left\|e^{|x|^{2} / \alpha^{2}} u(1)\right\|^{t}
$$

when $0 \leq t \leq 1$ and $0<\epsilon \leq \epsilon_{0}$ and with $N=N(\alpha, \beta)$. Then Lemma 4 gives

$$
\begin{aligned}
\| \sqrt{t(1-t)} e^{\gamma_{\epsilon}|x|^{2}} & \nabla \widetilde{u}_{\epsilon}\left\|_{L^{2}\left(\mathbb{R}^{n} \times[0,1]\right)}+\right\| \sqrt{t(1-t)}|x| e^{\gamma_{\epsilon}|x|^{2}} \nabla \widetilde{u}_{\epsilon} \|_{L^{2}\left(\mathbb{R}^{n} \times[0,1]\right)} \\
& \leq N e^{N\left(M_{1}+M_{2}(\epsilon)+M_{1}^{2}+M_{2}(\epsilon)^{2}\right)}\left[\left\|e^{|x|^{2} / \beta^{2}} u(0)\right\|+\left\|e^{|x|^{2} / \alpha^{2}} u(1)\right\|\right]
\end{aligned}
$$

when $0<\epsilon \leq \epsilon_{0}$, and the "logarithmic convexity" and regularity of $u$ follow from the limit of the identity in 4.11, the final limit relation between the variables $s$ and $t$, $s=\frac{\beta t}{\alpha(1-t)+\beta t}$, and letting $\epsilon$ tend to zero in 4.20 and in the above inequality.

Remark 1. We thank R. Killip for pointing out the following application of Lemma 1 and the identities (4.4) to generate Gaussian decaying solutions of $\partial_{t}=i H$ when $H=$ $\Delta+V_{1}(x)$ and $V_{1}$ satisfy the conditions in Theorem 3 In fact, if $e^{\gamma|x|^{2}} u_{0}$ is in $L^{2}\left(\mathbb{R}^{n}\right)$ and $u(t)=e^{i t H}\left(e^{H} u_{0}\right)$, we have $u(t)=e^{(1 / t+i) t H} u_{0}$, and from Lemma 1 ,

$$
\left\|e^{\frac{\gamma|x|^{2}}{1+4 \gamma\left(1+t^{2}\right)}} u(t)\right\| \leq e^{\left\|V_{1}\right\|_{\infty}}\left\|e^{\gamma|x|^{2}} u_{0}\right\| \quad \text { when } t \geq 0 .
$$

Next, we recall the following result established in [11]: 
Lemma 6. There are $N$ and $\epsilon_{0}>0$ such that the following holds: If $\lambda$ is in $\mathbb{R}^{n}, V$ is a complex-valued potential, $\|V\|_{L^{1}\left([0,1], L^{\infty}\left(\mathbb{R}^{n}\right)\right)} \leq \epsilon_{0}$ and $u \in C\left([0,1], L^{2}\left(\mathbb{R}^{n}\right)\right)$ satisfies

$$
\partial_{t} u=i(\Delta u+V(x, t) u+F(x, t)) \quad \text { in } \mathbb{R}^{n} \times[0,1],
$$

then

$$
\sup _{[0,1]}\left\|e^{\lambda \cdot x} u(t)\right\| \leq N\left[\left\|e^{\lambda \cdot x} u(0)\right\|+\left\|e^{\lambda \cdot x} u(1)\right\|+\left\|e^{\lambda \cdot x} F(t)\right\|_{L^{1}\left([0,1], L^{2}\left(\mathbb{R}^{n}\right)\right)}\right] .
$$

Theorem 5. Assume that $u$ in $C\left([0,1], L^{2}\left(\mathbb{R}^{n}\right)\right)$ satisfies

$$
\partial_{t} u=i(\Delta u+V(x, t) u) \quad \text { in } \mathbb{R}^{n} \times[0,1],
$$

where $V$ is in $L^{\infty}\left(\mathbb{R}^{n} \times[0,1]\right), \lim _{R \rightarrow+\infty}\|V\|_{L^{1}\left([0,1], L^{\infty}\left(\mathbb{R}^{n} \backslash B_{R}\right)\right)}=0, \alpha$ and $\beta$ are positive, and $\left\|e^{|x|^{2} / \beta^{2}} u(0)\right\|,\left\|e^{|x|^{2} / \alpha^{2}} u(1)\right\|$ are finite. Then there is $N=N(\alpha, \beta)$ such that

$$
\begin{aligned}
& \sup _{[0,1]}\left\|e^{\frac{|x|^{2}}{(\alpha t+(1-t) \beta)^{2}}} u(t)\right\|+\left\|\sqrt{t(1-t)} e^{\frac{|x|^{2}}{(\alpha t+(1-t) \beta)^{2}}} \nabla u\right\|_{L^{2}\left(\mathbb{R}^{n} \times[0,1]\right)} \\
& \quad \leq N e^{N \sup _{[0,1]}\|V(t)\|_{\infty}\left[\left\|e^{|x|^{2} / \beta^{2}} u(0)\right\|+\left\|e^{|x|^{2} / \alpha^{2}} u(1)\right\|+\sup _{[0,1]}\|u(t)\|\right]}
\end{aligned}
$$

Proof. Set $\gamma=1 / \alpha \beta$ and let

$$
\tilde{u}(x, t)=\left(\frac{\sqrt{\alpha \beta}}{\alpha(1-t)+\beta t}\right)^{n / 2} u\left(\frac{\sqrt{\alpha \beta} x}{\alpha(1-t)+\beta t}, \frac{\beta t}{\alpha(1-t)+\beta t}\right) e^{\frac{(\alpha-\beta)|x|^{2}}{4 i(\alpha(1-t)+\beta t)}}
$$

denote the function associated in Lemma 5 to $u$ when $A+i B=i$. This function is in $C\left([0,1], L^{2}\left(\mathbb{R}^{n}\right)\right)$ and satisfies

$$
\partial_{t} \tilde{u}=i(\Delta \tilde{u}+\tilde{V}(x, t) \tilde{u}) \quad \text { in } \mathbb{R}^{n} \times[0,1]
$$

with

$$
\begin{gathered}
\tilde{V}(x, t)=\frac{\alpha \beta}{(\alpha(1-t)+\beta t)^{2}} V\left(\frac{\sqrt{\alpha \beta} x}{\alpha(1-t)+\beta t}, \frac{\beta t}{\alpha(1-t)+\beta t}\right), \\
\sup _{[0,1]}\|\tilde{V}(t)\|_{\infty} \leq \max \left\{\frac{\alpha}{\beta}, \frac{\beta}{\alpha}\right\} \sup _{[0,1]}\|V(t)\|_{\infty}, \lim _{R \rightarrow+\infty}\|\tilde{V}\|_{L^{1}\left([0,1], L^{\infty}\left(\mathbb{R}^{n} \backslash B_{R}\right)\right)}=0,
\end{gathered}
$$

and

$$
\begin{aligned}
& \left\|e^{\gamma|x|^{2}} \tilde{u}(t)\right\|=\left\|e^{\frac{|x|^{2}}{(\alpha s+\beta(1-s))^{2}}} u(s)\right\|, \\
& \|\widetilde{u}(t)\|=\|u(s)\| \quad \text { when } \quad s=\frac{\beta t}{\alpha(1-t)+\beta t} .
\end{aligned}
$$

Choose $R>0$ such that $\|\widetilde{V}\|_{L^{1}\left([0,1], L^{\infty}\left(\mathbb{R}^{n} \backslash B_{R}\right)\right)} \leq \epsilon_{0}$. Then

$$
\partial_{t} \tilde{u}=i\left(\Delta \tilde{u}+\widetilde{V}_{R}(x, t) \tilde{u}+\widetilde{F}_{R}(x, t)\right)
$$


with $\widetilde{V}_{R}(x, t)=\chi_{\mathbb{R}^{n} \backslash B_{R}} \widetilde{V}(x, t), \widetilde{F}_{R}=\chi_{B_{R}} \widetilde{V}(x, t) \widetilde{u}$, and Lemma[6 yields

$$
\sup _{[0,1]}\left\|e^{\lambda \cdot x} \widetilde{u}(t)\right\| \leq N\left[\left\|e^{\lambda \cdot x} \widetilde{u}(0)\right\|+\left\|e^{\lambda \cdot x} \widetilde{u}(1)\right\|+e^{|\lambda| R} \sup _{[0,1]}\|\widetilde{V}(t)\|_{\infty} \sup _{[0,1]}\|\widetilde{u}(t)\|\right] .
$$

Replace $\lambda$ by $\lambda \sqrt{\gamma}$ in the above inequality, square both sides, multiply all by $e^{-|\lambda|^{2} / 2}$ and integrate both sides with respect to $\lambda$ in $\mathbb{R}^{n}$. This and the identity

$$
\int_{\mathbb{R}^{n}} e^{2 \sqrt{\gamma} \lambda \cdot x-|\lambda|^{2} / 2} d \lambda=(2 \pi)^{n / 2} e^{2 \gamma|x|^{2}}
$$

imply the inequality

$$
\sup _{[0,1]}\left\|e^{\gamma|x|^{2}} \widetilde{u}(t)\right\| \leq N\left[\left\|e^{\gamma|x|^{2}} \widetilde{u}(0)\right\|+\left\|e^{\gamma|x|^{2}} \widetilde{u}(1)\right\|+e^{2 \gamma R^{2}} \sup _{[0,1]}\|\widetilde{V}(t)\|_{\infty} \sup _{[0,1]}\|\widetilde{u}(t)\|\right] .
$$

This inequality and 4.22 imply that

$$
\begin{aligned}
& \sup _{[0,1]}\left\|e^{\gamma|x|^{2}} \widetilde{u}(t)\right\| \\
& \quad \leq N\left[\left\|e^{|x|^{2} / \beta^{2}} u(0)\right\|+\left\|e^{|x|^{2} / \alpha^{2}} u(1)\right\|+\sup _{[0,1]}\|V(t)\|_{\infty} \sup _{[0,1]}\|u(t)\|\right]
\end{aligned}
$$

for some new constant $N$.

To prove the regularity of $u$ we proceed as in $4.1-4.3$. The Duhamel formula shows that

$$
\widetilde{u}(t)=e^{i t \Delta} \widetilde{u}(0)+i \int_{0}^{t} e^{i(t-s) \Delta}(\widetilde{V}(s) \widetilde{u}(s)) d s \quad \text { in } \mathbb{R}^{n} \times[0,1] .
$$

For $0<\epsilon<1$, set

$$
\begin{aligned}
& \widetilde{F}_{\epsilon}(t)=\frac{i}{\epsilon+i} e^{\epsilon t \Delta}(\widetilde{V}(t) \widetilde{u}(t)), \\
& \widetilde{u}_{\epsilon}(t)=e^{(\epsilon+i) t \Delta} \widetilde{u}(0)+(\epsilon+i) \int_{0}^{t} e^{(\epsilon+i)(t-s) \Delta} \widetilde{F}_{\epsilon}(s) d s .
\end{aligned}
$$

The identities [14]

$$
e^{\left(z_{1}+z_{2}\right) \Delta}=e^{\left(z_{2}+z_{1}\right) \Delta}=e^{z_{1} \Delta} e^{z_{2} \Delta} \quad \text { when } \operatorname{Re} z_{1}, \operatorname{Re} z_{2} \geq 0,
$$

(4.24), (4.25) and 4.26) show that

$$
\tilde{u}_{\epsilon}(t)=e^{\epsilon t \Delta} \tilde{u}(t) \quad \text { when } 0 \leq t \leq 1,
$$

and from Lemma 1 with $A+i B=\epsilon$, 4.27) and 4.25,

$$
\begin{aligned}
& \sup _{[0,1]}\left\|e^{\gamma_{\epsilon}|x|^{2}} \widetilde{u}_{\epsilon}(t)\right\| \leq \sup _{[0,1]}\left\|e^{\gamma|x|^{2}} \widetilde{u}(t)\right\|, \\
& \sup _{[0,1]}\left\|e^{\gamma_{\epsilon}|x|^{2}} \widetilde{F}_{\epsilon}(t)\right\| \leq e^{\sup _{[0,1]}\|\widetilde{V}(t)\|_{\infty}} \sup _{[0,1]}\left\|e^{\gamma|x|^{2}} \widetilde{u}(t)\right\|,
\end{aligned}
$$


when $\gamma_{\epsilon}=\frac{\gamma}{1+4 \gamma \epsilon}$. Then Lemma 4, 4.28), and 4.23) show that

$$
\begin{aligned}
\left\|\sqrt{t(1-t)} e^{\gamma_{\epsilon}|x|^{2}} \nabla \widetilde{u}_{\epsilon}\right\|_{L^{2}\left(\mathbb{R}^{n} \times[0,1]\right)}+\left\|\sqrt{t(1-t)}|x| e^{\gamma_{\epsilon}|x|^{2}} \tilde{u}_{\epsilon}\right\|_{L^{2}\left(\mathbb{R}^{n} \times[0,1]\right)} \\
\leq N e^{N \sup _{[0,1]}\|V(t)\|_{\infty}}\left[\left\|e^{|x|^{2} / \beta^{2}} u(0)\right\|+\left\|e^{|x|^{2} / \alpha^{2}} u(1)\right\|+\sup _{[0,1]}\|u(t)\|\right] .
\end{aligned}
$$

The lemma follows from this inequality, 4.22), 4.23, , 4.21, and letting $\epsilon$ tend to zero.

\section{A Hardy type uncertainty principle. Proof of Theorem 1}

As we mentioned in the introduction, the motivation behind the Carleman inequality in Lemma 7 below is the following monotonicity or frequency function argument related to Lemma 2

If $u$ in $C\left([0,1], L^{2}\left(\mathbb{R}^{n}\right)\right)$ is a free solution to the free Schrödinger equation

$$
\partial_{t} u-i \Delta u=0 \quad \text { in } \mathbb{R}^{n} \times[0,1],
$$

$\left\|e^{\gamma|x|^{2}} u(0)\right\|,\left\|e^{\gamma|x|^{2}} u(1)\right\|$ are both finite, $f=e^{\mu\left|x+R t(1-t) e_{1}\right|^{2}-R^{2} t(1-t) / 8 \mu} u$ and $H=$ $(f, f)$, then $\log H$ is logarithmically convex in $[0,1]$ when $0<\mu<\gamma$.

The formal application of the above argument to a $C\left([0,1], L^{2}\left(\mathbb{R}^{n}\right)\right)$ solution to

$$
\partial_{t} u-i(\Delta u+V(x, t) u)=0 \quad \text { in } \mathbb{R}^{n} \times[0,1]
$$

implies a similar result when $V$ is a bounded potential, though the justification of the correctness of the manipulations involved in the corresponding formal application of Lemma 2 is not obvious to us. In fact, we can only justify these manipulations when the potential $V$ satisfies the first condition in Theorem 1 or when we can obtain the additional regularity of the gradient of $u$ in the strip, as in Theorem 5 . Here, we choose to prove Theorem 1 using the Carleman inequality in Lemma 7 in place of the above convexity argument. The reason for our choice is that it is simpler to justify the correctness of the application of the Carleman inequality to a $C\left([0,1], L^{2}\left(\mathbb{R}^{n}\right)\right)$ solution to (5.1) than the corresponding monotonicity or logarithmic convexity of the solution.

Lemma 7. The inequality

$$
\begin{aligned}
& R \sqrt{\frac{\epsilon}{8 \mu}}\left\|e^{\mu\left|x+R t(1-t) e_{1}\right|^{2}-(1+\epsilon) R^{2} t(1-t) / 16 \mu} g\right\|_{L^{2}\left(\mathbb{R}^{n+1}\right)} \\
& \leq\left\|e^{\mu\left|x+R t(1-t) e_{1}\right|^{2}-(1+\epsilon) R^{2} t(1-t) / 16 \mu}\left(\partial_{t}-i \Delta\right) g\right\|_{L^{2}\left(\mathbb{R}^{n+1}\right)}
\end{aligned}
$$

holds when $\epsilon>0, \mu>0, R>0$, and $g \in C_{0}^{\infty}\left(\mathbb{R}^{n+1}\right)$. 
Proof. Let $f=e^{\mu\left|x+R t(1-t) e_{1}\right|^{2}-(1+\epsilon) R^{2} t(1-t) / 16 \mu} g$. Then

$$
e^{\mu\left|x+R t(1-t) e_{1}\right|^{2}-(1+\epsilon) R^{2} t(1-t) / 16 \mu}\left(\partial_{t}-i \Delta\right) g=\partial_{t} f-\mathcal{S} f-\mathcal{A} f
$$

and from 2.12-2.14 with $\gamma=1, A+i B=i$ and

$$
\varphi(x, t)=\mu\left|x+R t(1-t) e_{1}\right|^{2}-\frac{(1+\epsilon) R^{2} t(1-t)}{16 \mu},
$$

we have

$$
\begin{gathered}
\mathcal{S}=-4 \mu i\left(x+\operatorname{Rt}(1-t) e_{1}\right) \cdot \nabla-2 \mu n i \\
+2 \mu R(1-2 t)\left(x_{1}+R t(1-t)\right)-\frac{(1+\epsilon) R^{2}(1-2 t)}{16 \mu}, \\
\mathcal{A}=i \Delta+4 \mu^{2} i\left|x+\operatorname{Rt}(1-t) e_{1}\right|^{2}, \\
\mathcal{S}_{t}+[\mathcal{S}, \mathcal{A}]=-8 \mu \Delta+32 \mu^{3}\left|x+R t(1-t) e_{1}\right|^{2}-4 \mu R\left(x_{1}+R t(1-t)\right) \\
+2 \mu R^{2}(1-2 t)^{2}+\frac{(1+\epsilon) R^{2}}{8 \mu}-4 i \mu R(1-2 t) \partial_{x_{1}}
\end{gathered}
$$

and

$$
\begin{aligned}
\left(\mathcal{S}_{t} f+[\mathcal{S}, \mathcal{A}] f, f\right)= & 32 \mu^{3} \int\left|x+R t(1-t) e_{1}-\frac{R}{16 \mu^{2}} e_{1}\right|^{2}|f|^{2} d x+\frac{\epsilon R^{2}}{8 \mu} \int|f|^{2} d x \\
& +8 \mu \int\left|\nabla_{x^{\prime}} f\right|^{2} d x+8 \mu \int\left|i \partial_{x_{1}} f-\frac{R(1-2 t)}{2} f\right|^{2} d x \\
\geq & \frac{\epsilon R^{2}}{8 \mu} \int|f|^{2} d x .
\end{aligned}
$$

Following the standard method to handle $L^{2}$-Carleman inequalities [6], the symmetric and skew-symmetric parts of $\partial_{t}-\mathcal{S}-\mathcal{A}$, as a space-time operator, are respectively $-\mathcal{S}$ and $\partial_{t}-\mathcal{A}$, and its space-time commutator $\left[-\mathcal{S}, \partial_{t}-\mathcal{A}\right]$ is $\mathcal{S}_{t}+[\mathcal{S}, \mathcal{A}]$. Thus,

$$
\begin{aligned}
& \| \partial_{t} f- \mathcal{S} f-\mathcal{A} f \|_{L^{2}\left(\mathbb{R}^{n+1}\right)}^{2} \\
& \quad=\left\|\partial_{t} f-\mathcal{A} f\right\|_{L^{2}\left(\mathbb{R}^{n+1}\right)}^{2}+\|\mathcal{S} f\|_{L^{2}\left(\mathbb{R}^{n+1}\right)}^{2}-2 \operatorname{Re} \iint \mathcal{S} f \overline{\partial_{t} f-\mathcal{A} f} d x d t \\
& \quad \geq \iint\left[-\mathcal{S}, \partial_{t}-\mathcal{A}\right] f \bar{f} d x d t=\int\left(\mathcal{S}_{t} f+[\mathcal{S}, \mathcal{A}] f, f\right) d t,
\end{aligned}
$$

and the lemma follows from 5.3 and 5.2 .

Proof of Theorem 1 . Let $u$ be as in Theorem 1 and $\tilde{u}, \tilde{V}$ the corresponding functions defined in Lemma 5 when $A+i B=i$. Then $\tilde{u}$ is in $C\left([0,1], L^{2}\left(\mathbb{R}^{n}\right)\right)$,

$$
\partial_{t} \tilde{u}=i(\Delta \widetilde{u}+\widetilde{V}(x, t) \widetilde{u}) \quad \text { in } \mathbb{R}^{n} \times[0,1],
$$


$\left\|e^{\gamma|x|^{2}} \widetilde{u}(0)\right\|,\left\|e^{\gamma|x|^{2}} \widetilde{u}(1)\right\|$ are finite for $\gamma=1 / \alpha \beta$ and $\gamma>1 / 2$. The proofs of Theorem 3 or 5 show that in either case

$$
N_{\gamma}=\sup _{[0,1]}\left\|e^{\gamma|x|^{2}} \widetilde{u}(t)\right\|+\left\|\sqrt{t(1-t)} e^{\gamma|x|^{2}} \nabla \widetilde{u}\right\|_{L^{2}\left(\mathbb{R}^{n} \times[0,1]\right)}<+\infty .
$$

For given $R>0$, choose $\mu$ and $\epsilon$ such that

$$
\frac{(1+\epsilon)^{3 / 2}}{2(1-\epsilon)^{3}}<\mu \leq \frac{\gamma}{1+\epsilon}
$$

and let $\theta_{M}$ and $\eta_{R}$ be smooth functions satisfying $\theta_{M}(x)=1$ when $|x| \leq M, \theta_{M}(x)=0$ when $|x|>2 M, M \geq R, \eta_{R} \in C_{0}^{\infty}(0,1), 0 \leq \eta_{R} \leq 1, \eta_{R}(t)=1$ in $[1 / R, 1-1 / R]$ and $\eta_{R}=0$ in $[0,1 / 2 R] \cup[1-1 / 2 R, 1]$. Then

$$
g(x, t)=\theta_{M}(x) \eta_{R}(t) \widetilde{u}(x, t)
$$

is compactly supported in $\mathbb{R}^{n} \times(0,1)$ and

$$
\partial_{t} g-i(\Delta g+\widetilde{V} g)=\theta_{M} \eta_{R}^{\prime} \widetilde{u}-i\left(2 \nabla \theta_{M} \cdot \nabla \widetilde{u}+\widetilde{u} \Delta \theta_{M}\right) \eta_{R} .
$$

The first term on the right-hand side of (5.6) is supported where

$$
\mu\left|x+R t(1-t) e_{1}\right|^{2} \leq \mu(1+\epsilon)|x|^{2}+\mu(1+1 / \epsilon) \leq \gamma|x|^{2}+\gamma / \epsilon,
$$

and the second inside $B_{2 M} \backslash B_{M} \times[1 / 2 R, 1-1 / 2 R]$, where

$$
\mu\left|x+R t(1-t) e_{1}\right|^{2} \leq \gamma|x|^{2}+\gamma R^{2} / \epsilon .
$$

Apply now Lemma 7 to $g$ with the values of $\mu$ and $\epsilon$ chosen in (5.5). This, the bounds for $\mu\left|x+R t(1-t) e_{1}\right|^{2}$ in each of the parts of the support of $\partial_{t} g-i(\Delta g+\widetilde{V} g)$, and the natural bounds for $\nabla \theta_{M}, \Delta \theta_{M}$, and $\eta_{R}^{\prime}$ show that there is a constant $N_{\epsilon}$ such that

$$
\begin{aligned}
& R\left\|e^{\mu\left|x+R t(1-t) e_{1}\right|^{2}-(1+\epsilon) R^{2} t(1-t) / 16 \mu} g\right\|_{L^{\infty}\left(\mathbb{R}^{n} \times[0,1]\right)} \\
& \leq N_{\epsilon}\|\widetilde{V}\|_{L^{\infty}\left(\mathbb{R}^{n} \times[0,1]\right)}\left\|e^{\mu\left|x+R t(1-t) e_{1}\right|^{2}-(1+\epsilon) R^{2} t(1-t) / 16 \mu} g\right\|_{L^{2}\left(\mathbb{R}^{n} \times[0,1]\right)} \\
& +N_{\epsilon} R e^{\gamma / \epsilon} \sup _{[0,1]}\left\|e^{\gamma|x|^{2}} \widetilde{u}(t)\right\| \\
& +N_{\epsilon} M^{-1} e^{\gamma R^{2} / \epsilon}\left\|e^{\gamma|x|^{2}}(|\widetilde{u}|+|\nabla \widetilde{u}|)\right\|_{L^{2}\left(\mathbb{R}^{n} \times[1 / 2 R, 1-1 / 2 R]\right)} .
\end{aligned}
$$

The first term on the right-hand side of (5.7) can be hidden in the left-hand side when $R \geq 2 N_{\epsilon}\|\widetilde{V}\|_{L^{\infty}\left(\mathbb{R}^{n} \times[0,1]\right)}$, while the last one tends to zero when $M$ tends to infinity by (5.4). This and the fact that $g=\widetilde{u}$ in $B_{\epsilon(1-\epsilon)^{2} R / 4} \times[(1-\epsilon) / 2,(1+\epsilon) / 2]$, where

$$
\mu\left|x+R t(1-t) e_{1}\right|^{2}-\frac{(1+\epsilon) R^{2} t(1-t)}{16 \mu} \geq \frac{R^{2}}{16 \mu}\left(4 \mu^{2}(1-\epsilon)^{6}-(1+\epsilon)^{3}\right),
$$


and 5.5) show that

$$
R e^{C(\gamma, \epsilon) R^{2}}\|\widetilde{u}\|_{L^{2}\left(B_{R / 8} \times[(1-\epsilon) / 2,(1+\epsilon) / 2]\right)} \leq N_{\gamma, \epsilon} R
$$

when $R \geq 2 N_{\epsilon}\|\widetilde{V}\|_{L^{\infty}\left(\mathbb{R}^{n} \times[0,1]\right)}$. At the same time,

$$
N^{-1}\|\tilde{u}(0)\| \leq\|\widetilde{u}(t)\| \leq N\|\widetilde{u}(0)\| \quad \text { when } 0 \leq t \leq 1, \quad N=e^{\sup _{[0,1]}\|\operatorname{Im} \tilde{V}(t)\|_{\infty},}
$$

and from (5.4),

$$
\|\widetilde{u}(t)\| \leq\|\widetilde{u}(t)\|_{L^{2}\left(B_{R / 8}\right)}+e^{-\gamma R^{2} / 64} N_{\gamma} \quad \text { when } 0 \leq t \leq 1 .
$$

Then 5.8-5.10) show that there is a constant $N_{\gamma, \epsilon, V}$, which depends on $N_{\gamma}, \epsilon$ and $\sup _{[0,1]}\|V(t)\|_{\infty}$, such that

$$
e^{C(\gamma, \epsilon) R^{2}}\|\widetilde{u}(0)\| \leq N_{\gamma, \epsilon, V} .
$$

Let then $R$ tend to infinity to derive that $u \equiv 0$.

\section{A positive commutator and a misleading frequency function}

If $f=e^{a(t)|x|^{2}} u$ and $u$ is a solution to the free Schrödinger equation in $\mathbb{R} \times[-1,1]$, then $f$ satisfies $\partial_{t} f=\mathcal{S} f+\mathcal{A} f$ with symmetric and skew-symmetric operators

$$
\mathcal{S}=-4 i a\left(x \partial_{x}+1 / 2\right)+a^{\prime} x^{2}, \quad \mathcal{A}=i\left(\partial_{x}^{2}+4 a^{2} x^{2}\right) .
$$

In this case (see 2.14)

$$
\mathcal{S}_{t}+[\mathcal{S}, \mathcal{A}]=\left(2 a^{\prime} / a\right) \mathcal{S}-8 a \partial_{x}^{2}+\left(32 a^{3}+a^{\prime \prime}-2 a^{\prime 2} / a\right) x^{2},
$$

and if $a$ is a positive and even solution of

$$
32 a^{3}+a^{\prime \prime}-2 a^{\prime 2} / a=0 \quad \text { in }[-1,1],
$$

the formal calculations in Lemma 2 show that $H_{a}(t)=\left\|e^{a(t) x^{2}} u(t)\right\|^{2}$ satisfies

$$
\partial_{t}\left(a^{-2} \partial_{t} \log H_{a}(t)\right) \geq 0 \quad \text { in }[-1,1],
$$

and the integration of the inequality

$$
a(\tau)^{2} \partial_{s} \log H_{a}(s) \leq a(s)^{2} \partial_{\tau} \log H_{a}(s) \quad \text { when }-1 \leq s \leq 0 \leq \tau \leq 1
$$

implies that

$$
H_{a}(0) \leq H_{a}(-1)^{1 / 2} H_{a}(1)^{1 / 2} .
$$

On the other hand, if $a$ solves

$$
\left\{\begin{array}{l}
32 a^{3}+a^{\prime \prime}-2 a^{\prime 2} / a=0 \\
a(0)=1, \quad a^{\prime}(0)=0
\end{array}\right.
$$


then $a$ is positive, even and $\lim _{R \rightarrow+\infty} R a(R)=0$. Moreover, $a_{R}(t)=R a(R t)$ also solves 6.1], and if the formal calculation is correct for $H_{a_{R}}$, 6.2) would imply that

$$
\left\|e^{R x^{2}} u(0)\right\|^{2} \leq\left\|e^{R a(R) x^{2}} u(-1)\right\|\left\|e^{R a(R) x^{2}} u(1)\right\| .
$$

In particular, $u \equiv 0$; but

$$
u(x, t)=(t-i)^{-1 / 2} e^{\frac{i|x|^{2}}{4(t-i)}}
$$

contradicts this.

This shows that there are functions $\varphi$ which make non-negative the commutator of the symmetric and skew-symmetric parts of $e^{\varphi}\left(\partial_{t}-i \partial_{x}^{2}\right) e^{-\varphi}$ and such that it is not possible to plug in or enter in the associated Carleman inequality or frequency function some reasonable solutions of the free Schrödinger equation. It also shows that the rather complex arguments we used to derive the logarithmic convexity of

$$
H(t)=\int_{\mathbb{R}^{n}} e^{2 \gamma|x|^{2}}|u(t)|^{2} d x
$$

are in fact necessary when $u$ in $C\left([0,1], L^{2}\left(\mathbb{R}^{n}\right)\right)$ is a solution satisfying the conditions in Lemma 3 until a more suitable representation formula for these solutions is available. By suitable we mean a formula which allows deriving the quadratic exponential decay of the solution in the interior of a time slab from the known decay of the solution at the top and bottom of the slab.

\section{Parabolic analog. Proof of Theorem 4}

Assume that $u$ satisfies the conditions in Theorem 4 and let $\widetilde{u}$ be the conformal or Appell transformation of $u$ defined in Lemma $[5$ with $A+i B=1, \alpha=1$ and $\beta=1+2 / \delta$. Then $\widetilde{u}$ is in $L^{\infty}\left([0,1], L^{2}\left(\mathbb{R}^{n}\right)\right) \cap L^{2}\left([0, T], H^{1}\left(\mathbb{R}^{n}\right)\right)$, satisfies

$$
\partial_{t} \tilde{u}=\Delta \widetilde{u}+\widetilde{V}(x, t) \widetilde{u} \quad \text { in } \mathbb{R}^{n} \times(0,1]
$$

with $\widetilde{V}$ a bounded potential in $\mathbb{R}^{n} \times[0,1]$, and if $\gamma=1 / 2 \delta$, we have

$$
\left\|e^{\gamma|x|^{2}} \widetilde{u}(0)\right\|=\|u(0)\|, \quad\left\|e^{\gamma|x|^{2}} \widetilde{u}(1)\right\|=\left\|e^{|x|^{2} / \delta^{2}} u(1)\right\| .
$$

From Lemmas 3 and 4 with $A+i B=1$, we have

$$
\begin{aligned}
\sup _{[0,1]}\left\|e^{\gamma|x|^{2}} \widetilde{u}(t)\right\|+\left\|\sqrt{t(1-t)} e^{\gamma|x|^{2}} \nabla \widetilde{u}\right\|_{L^{2}\left(\mathbb{R}^{n} \times[0,1]\right)} & \\
\leq & e^{N\left(M_{1}+M_{1}^{2}\right)}\left(\left\|e^{\gamma|x|^{2}} \widetilde{u}(0)\right\|+\left\|e^{\gamma|x|^{2}} \widetilde{u}(1)\right\|\right),
\end{aligned}
$$

where $M_{1}=\|\tilde{V}\|_{L^{\infty}\left(\mathbb{R}^{n} \times[0,1]\right)}$. The proof is finished by plugging in

$$
g(x, t)=\theta_{M}(x) \eta_{R}(t) \widetilde{u}(x, t)
$$

in the Carleman inequality below and in complete analogy with the argument we used to prove Theorem 1 . 
Lemma 8. The inequality

$$
\begin{aligned}
& R \sqrt{\frac{\epsilon}{8 \mu}}\left\|e^{\mu\left|x+R t(1-t) e_{1}\right|^{2}+R^{2} t(1-t)(1-2 t) / 6-(1+\epsilon) R^{2} t(1-t) / 16 \mu} g\right\|_{L^{2}\left(\mathbb{R}^{n+1}\right)} \\
& \quad \leq\left\|e^{\mu\left|x+R t(1-t) e_{1}\right|^{2}+R^{2} t(1-t)(1-2 t) / 6-(1+\epsilon) R^{2} t(1-t) / 16 \mu}\left(\partial_{t}-\Delta\right) g\right\|_{L^{2}\left(\mathbb{R}^{n+1}\right)}
\end{aligned}
$$

holds when $\epsilon>0, \mu>0, R>0$, and $g \in C_{0}^{\infty}\left(\mathbb{R}^{n+1}\right)$.

Proof. Let $f=e^{\mu\left|x+R t(1-t) e_{1}\right|^{2}+R^{2} t(1-t)(1-2 t) / 6-(1+\epsilon) R^{2} t(1-t) / 16 \mu} g$. Then

$$
e^{\mu\left|x+R t(1-t) e_{1}\right|^{2}+R^{2} t(1-t)(1-2 t) / 6-(1+\epsilon) R^{2} t(1-t) / 16 \mu}\left(\partial_{t}-\Delta\right) g=\partial_{t} f-\mathcal{S} f-\mathcal{A} f,
$$

and from 2.12 -2.14 with $\gamma=1, A+i B=1$ and

$$
\varphi(x, t)=\mu\left|x+R t(1-t) e_{1}\right|^{2}+R^{2} t(1-t)(1-2 t) / 6-(1+\epsilon) R^{2} t(1-t) / 16 \mu,
$$

we have

$$
\begin{aligned}
\mathcal{S}= & \Delta+4 \mu^{2}\left|x+R t(1-t) e_{1}\right|^{2}+2 \mu R(1-2 t)\left(x_{1}+R t(1-t)\right) \\
& +\left(t^{2}-t+1 / 6\right) R^{2}-(1+\epsilon) R^{2}(1-2 t) / 16 \mu, \\
\mathcal{A}= & -4 \mu\left(x+R t(1-t) e_{1}\right) \cdot \nabla-2 \mu n, \\
\mathcal{S}_{t}+[\mathcal{S}, \mathcal{A}]= & -8 \mu \Delta+32 \mu^{3}\left|x+R t(1-t) e_{1}\right|^{2}+2 \mu R^{2}(1-2 t)^{2} \\
& +4 \mu R(4 \mu(1-2 t)-1)\left(x_{1}+R t(1-t)\right)+(2 t-1) R^{2}+(1+\epsilon) R^{2} / 8 \mu
\end{aligned}
$$

and

$$
\begin{aligned}
\left(\mathcal{S}_{t} f+[\mathcal{S}, \mathcal{A}] f, f\right)= & 32 \mu^{3} \int\left|x+R t(1-t) e_{1}+\frac{(4 \mu(1-2 t)-1) R}{16 \mu^{2}} e_{1}\right|^{2}|f|^{2} d x \\
& +\mu \int|\nabla f|^{2} d x+\frac{\epsilon R^{2}}{8 \mu} \int|f|^{2} d x \\
\geq & \frac{\epsilon R^{2}}{8 \mu} \int|f|^{2} d x .
\end{aligned}
$$

Finally,

$$
\varphi(x, 1 / 2)=\mu\left|x+\frac{R}{4} e_{1}\right|^{2}-\frac{(1+\epsilon) R^{2}}{64 \mu} \geq\left(4 \mu^{2}(1-\epsilon)^{2}-(1+\epsilon)\right) \frac{R^{2}}{64 \mu}
$$

when $|x| \leq \epsilon R / 4$, and it is positive for $\mu>1 / 2$ and $\epsilon>0$ small.

Remark 2. 7.1), (7.2), and the interior regularity of parabolic equations show that the formal calculations in Lemma 2 to prove the logarithmic convexity of

$$
H_{\mu}(t)=\int_{\mathbb{R}^{n}} e^{2 \mu\left|x+R t(1-t) e_{1}\right|^{2}+R^{2} t(1-t)(1-2 t) / 3-R^{2} t(1-t) / 8 \mu}|\widetilde{u}(t)| d x
$$


are correct when $\mu<\gamma$. In particular,

$$
H_{\mu}(1 / 2) \leq e^{N\left(M_{1}+M_{1}^{2}\right)} H_{\mu}(0)^{1 / 2} H_{\mu}(1)^{1 / 2},
$$

and letting $\mu$ increase to $\gamma$ and then $R$ tend to infinity, one also finds that $\widetilde{u} \equiv 0$ in $\mathbb{R}^{n} \times[0,1]$ when $\gamma>1 / 2$.

Acknowledgments. The first and fourth authors are supported by MEC grant MTM2004-03029, the second and third authors by NSF grants DMS-0456583 and DMS-0456833 respectively.

\section{References}

[1] Bonami, A., Demange, B.: A survey on uncertainty principles related to quadratic forms. Collect. Math. 2006, Vol. Extra, 1-36 Zbl 1107.30021 MR 2264204

[2] Escauriaza, L., Kenig, C. E., Ponce, G., Vega, L.: On uniqueness properties of solutions of Schrödinger equations. Comm. Partial Differential Equations 31, 1811-1823 (2006) Zbl 1124.35068 MR 2273975

[3] Escauriaza, L., Kenig, C. E., Ponce, G., Vega, L.: On uniqueness properties of solutions of the $k$-generalized KdV. J. Funct. Anal. 244, 504-535 (2007) Zbl 1122.35124 MR 2297033

[4] Escauriaza, L., Kenig, C. E., Ponce, G., Vega, L.: Convexity of free solutions of Schrödinger equations with Gaussian decay. To appear

[5] Evans, L. C.: Partial Differential Equations. Amer. Math. Soc. (1998) Zbl 0902.35002 MR 1625845

[6] Hörmander, L.: Linear Partial Differential Operators. Springer, Berlin (1969) Zbl 0175.39201 MR 0248435

[7] Ionescu, A. D., Kenig, C. E.: $L^{p}$-Carleman inequalities and uniqueness of solutions of nonlinear Schrödinger equations. Acta Math. 193, 193-239 (2004) Zbl pre02183718 MR 2134866

[8] Ionescu, A. D., Kenig, C. E.: Uniqueness properties of solutions of Schrödinger equations. J. Funct. Anal. 232, 90-136 (2006) Zbl 1092.35104 MR 2200168

[9] Isakov, V.: Carleman type estimates in an anisotropic case and applications. J. Differential Equations 105, 217-238 (1993) Zbl 0851.35028 MR 1240395

[10] Jaming, P.: Uncertainty principles for orthonormal bases. arxiv.org/pdf/math/0606396

[11] Kenig, C. E., Ponce, G., Vega, L.: On unique continuation for nonlinear Schrödinger equations. Comm. Pure Appl. Math. 60, 1247-1262 (2002) Zbl 1041.35072 MR 1980854

[12] Kenig, C. E., Ponce, G., Vega, L.: On unique continuation of solutions to the generalized KdV equation. Math. Res. Lett. 10, 833-846 (2003) Zbl 1055.35102 MR 2025059

[13] Lions, J.-L., Malgrange, B.: Sur l'unicité rétrograde dans les problèmes mixtes paraboliques. Math. Scand. 8, 277-286 (1960) Zbl 0126.12202 MR 0140855

[14] Pazy, A.: Semigroups of Linear Operators with Application to Partial Differential Equations. Springer, Berlin (1983) Zbl 0516.47023 MR 0710486

[15] Sitaram, A., Sundari, M., Thangavelu, S.: Uncertainty principles on certain Lie groups. Proc. Indian Acad. Sci. Math. Sci. 105, 135-151 (1995) Zbl 0857.43011 MR 1350473

[16] Stein, E. M., Shakarchi, R.: Complex Analysis. Princeton Lectures in Analysis II, Princeton Univ. Press. (2003) Zbl 1020.30001 MR 1976398

[17] Thangavelu, S.: Lectures on Hermite and Laguerre Expansions. Princeton Univ. Press, Princeton, NJ (1993) Zbl 0791.41030 MR 1215939 